Check for updates

Cite this: Mater. Chem. Front., $2021,5,60$

Received 25th August 2020,

Accepted 17th September 2020

DOI: 10.1039/d0qm00626b

rsc.li/frontiers-materials

\title{
Recent advances in oligomers/polymers with unconventional chromophores
}

\begin{abstract}
Nan Jiang, ${ }^{a}$ Dongxia Zhu, (D) *a Zhongmin Su (D) *a and Martin R. Bryce (D) *b
Nonconventional chromophores, without classic conjugation and rigid units, are a hot topic of luminescent materials nowadays because of their unique under-explored fundamental properties and their promising applications. Despite the increased attention recently paid to this exciting area, the structural design and the mechanism of photoluminescence in these materials remain unclear and controversial. Different luminescence mechanisms have been successively proposed in recent years, such as oxidation, aggregation of carbonyl units, interactions with phenyl groups, clustering of chromophores and through-space conjugation, etc. This review summarizes oligomers/polymers with unconventional chromophores reported in recent years, which are classified according to their chemical structures and luminescence mechanisms. We present an interconnected overview of the topic highlighting the opportunities for new materials synthesis, looking forward to accelerated future innovation and developments in this area.
\end{abstract}

\section{Introduction}

In the past decades, organic photoluminescence (PL) materials have undergone continued experimental and theoretical development owing to their established applications in the fields of

\footnotetext{
${ }^{a}$ Key Laboratory of Nanobiosensing and Nanobioanalysis at Universities of Jilin Province, Department of Chemistry, Northeast Normal University, 5268 Renmin Street, Changchun, Jilin Province 130024, P. R. China. E-mail: zhudx047@nenu.edu.cn, zmsu@nenu.edu.cn

${ }^{b}$ Department of Chemistry, Durham University, Durham, DH1 3LE, UK. E-mail:m.r.bryce@durham.ac.uk
}

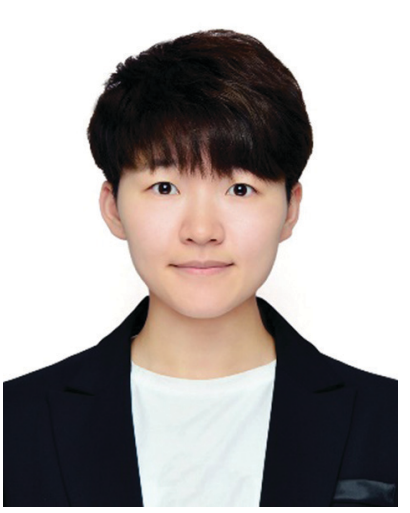

Nan Jiang
Nan Jiang received her BS degree from Tonghua Normal College in 2016. She is currently studying for her PhD degree at Northeast Normal University under Prof. Dong-Xia Zhu. And she is currently a joint PhD student at Durham University (UK) under the supervision of Prof. Martin R. Bryce. Her research is mainly on the development of functional polymeric photoelectric materials and intelligent response-type gel materials. optoelectronic devices, chemo-/bio-probes, solar cells, environmental sensors, etc. ${ }^{1-11}$ Traditional organic PL materials generally comprise predominantly $\pi$-conjugated units, such as phenyl, thiophene, fluorene, and carbazole. ${ }^{12-16}$ Although they have been exploited for many years, they still have some inherent disadvantages, including complex multi-step synthetic processes. Moreover, $\pi-\pi$ conjugated structures can limit many biological applications due to high cytotoxicity and immunogenicity; they generally exhibit aggregation caused quenching (ACQ) behavior, showing no emission in concentrated solution and solid states, which blocks some practical applications. ${ }^{17-19}$ These undesirable features have prompted an alternative design of some

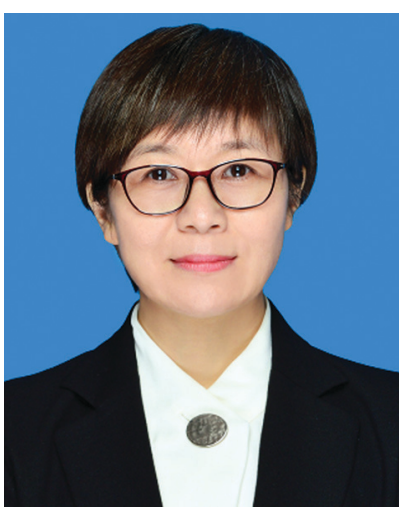

Dongxia Zhu
Prof. Dong-Xia Zhu received her $P h D$ (2005) in chemistry department under the supervision of Prof. Zhong-Min Su from NENU. In 2001-2002, she was a joint PhD student at Jilin University under the supervision of Prof. Yue Wang. In 2011-2012, she was an academic visitor at Durham University (UK) in the group of Prof. Martin R. Bryce and Prof. Todd B. Marder. She has been a full professor in chemistry department at NENU since 2017. Her research interests mainly focus on functional organic/polymeric photoelectric materials and devices. 
unorthodox luminescent materials that might overcome the above drawbacks. ${ }^{20-22}$ More recently, a growing body of research demonstrates that some materials without any typical chromophores in their structures, ${ }^{23-36}$ some even free of any (hetero)cyclic aromatic building blocks, ${ }^{37-41}$ can also be induced to produce intrinsic fluorescence and/or phosphorescence under certain conditions. Generally, these unconventional chromophores involve subgroups such as amino (-NH-), ${ }^{42}$ acylamino $(-\mathrm{NH}-\mathrm{CO}-),{ }^{43}$ phosphonate $\left(-\mathrm{P}(\mathrm{O})(\mathrm{OR})_{2}\right),{ }^{44}$ carbamate $(-\mathrm{NH}-$ COO- $),{ }^{45}$ sulfide $(-\mathrm{S}-)$, sulfoxide $(\mathrm{S}=\mathrm{O})$, sulfonyl $(\mathrm{O}=\mathrm{S}=\mathrm{O}),{ }^{37}$ cyano $(-\mathrm{C} \equiv \mathrm{N}),{ }^{39}$ alkene $(-\mathrm{C}=\mathrm{C}-),{ }^{46}$ hydroxyl $(-\mathrm{OH}),{ }^{47}$ ether $(-\mathrm{O}-),{ }^{48}$ etc, and some units that combine these subgroups, like anhydride, imide, sulfonate, and so forth. ${ }^{2,49}$ Among these emerging chromophores, oligomers/polymers have aroused great attention because of their advantages of better hydrophilicity, large structural diversity, low-cost and facile synthesis, high chain flexibility (easy to functionalize or form a supramolecular assembly) and good biocompatibility. ${ }^{22,50}$

Although these novel materials are making rapid progress, the understanding of the PL mechanism has not been unified, due to the diversity of atypical chromophores in the molecular structures and the complexity of the PL behavior. Various PL mechanisms have been proposed based on different systems, for example, oxidation/acidification, ${ }^{51,52}$ topological structure and effect of end groups, ${ }^{53}$ aggregation of carbonyl units, ${ }^{38}$ formation of hydrogen bonds, spatial electron delocalization, etc. ${ }^{54,55}$ On the one hand, it was initially thought that these materials which lack standard conjugated chromophores, could not be emitting, and that the observed PL originated from trace impurities in the material. On the other hand, with only limited understanding of the PL of such chromophores, researchers have often studied their photophysical properties under a restricted set of specific conditions. Therefore, lacking systematic investigation under different conditions means that many studies have failed to reach a comprehensive conclusion.

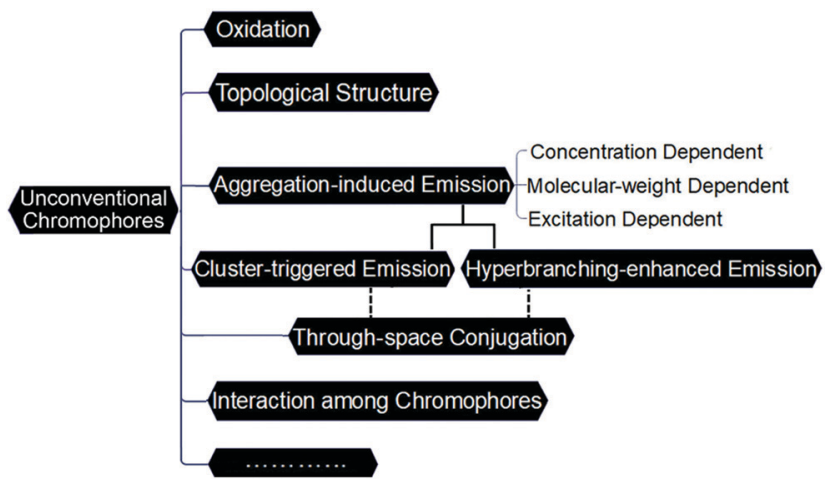

Scheme 1 An interconnected map of the ongoing understanding key features of the luminescence mechanisms of unconventional chromophores.

Scheme 1 shows a profile of the ongoing understanding of the luminescence mechanisms of nonconventional systems.

In the early stages, the atypical luminescent chromophores incorporated aliphatic amines [mainly composed of poly(amido amine)s (PAMAM)s] and it was recognized that the tertiary amines formed fluorescent centers after oxidation. Subsequently, new fluorescent systems were reported. ${ }^{51,56,57}$ These systems have two distinctive features: (i) they do not contain tertiary amine groups (or any $\mathrm{N}$ atoms) but all contain carbonyl (or ester) groups. (ii) No emission can be detected in dilute solution, while obvious fluorescence is observed in solid and viscous liquid states, which is similar to the aggregation-induced emission (AIE) materials that were a hot new research topic at that time.

In 2013, Yuan et al. found that ordinary rice emits strong blue light when exposed to ultraviolet light, which was later termed a clustering-triggered emission (CTE) mechanism and was categorized as an AIE system. ${ }^{58}$ In this concept, atypical chromophores aggregate into clusters, inducing effective short contacts, leading to the overlap of intra- and/or intermolecular electron clouds, bringing rigid molecular conformations and

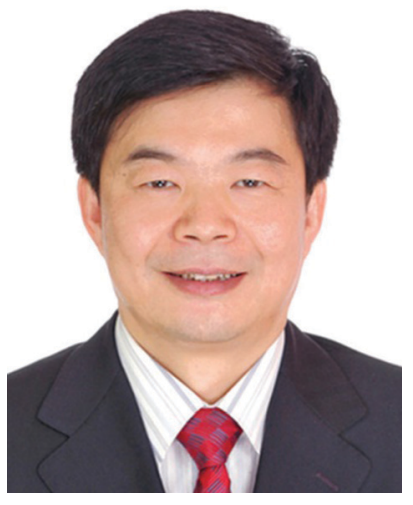

Zhongmin Su
Prof. Zhong-Min Su received his BS (1983) and then his PhD in inorganic chemistry under the supervision of Prof. Rong-Shun Wang and Prof. Chi-Ming Che from NENU (1997). He has been a full professor at NENU since 1994. Later, he worked as a visiting scholar in the group of Prof. Chi-Ming Che and Prof. Guan-Hua Chen (The University of Hong Kong), in Prof. Koji Ohta's group (National Industrial Technology Research Institute,

Japan), and in Prof. N. Roesch's group (Technical University of Munich, Germany). His research interests focus on functional material chemistry and quantum chemistry.

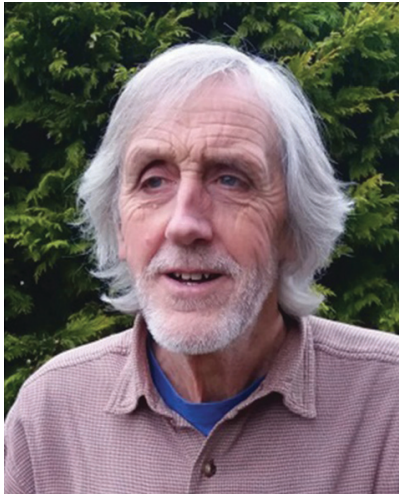

Martin R. Bryce
Prof. Martin Bryce received his BSc from Wolverhampton Polytechnic and his DPhil from the University of York, UK. After postdoctoral work at the University of British Columbia in Prof. Larry Weiler's group, and Bristol University in Prof. Roger Alder's group, he moved to Durham University. Since 1995 he has been a full professor of chemistry at Durham. He has held visiting scientist positions at the University of California, Santa Barbara, the University of Copenhagen and the University of Florida, Gainesville. His research covers molecular design, synthesis and properties of optoelectronic materials (small molecules and polymers), molecular electronics and nano-scale chemistry. 
extended conjugation, finally boosting the emission. Recently, another kind of AIE effect, hyperbranching-enhanced-emission (HEE) was also proposed. According to current understanding, two new categories of AIE, namely CTE and HEE, are triggered by through-space conjugation (TSC) of clusters of subgroups which strongly restrict non-radiative relaxation. These systems generally possess excitation-dependent, molecular weightdependent and concentration-dependent behaviors (Scheme 1). Atypical luminescent materials can emit light not only by clustering and conformational hardening at a relatively high concentration, but also by molecular aggregation at a relatively low concentration, showing AIE properties. Both the concentration enhanced PL and AIE properties are determined by the specific structural characteristics of the materials. A detailed analysis of structure-PL property relationships is necessary for a deeper and thorough understanding of the AIE mechanism of unconventional luminogens.

In the last few years, other molecular interactions leading to atypical PL have also been established, such as hydrogen bonds and ionic bonds, which are good for conformational rigidification, thus benefiting luminescence. ${ }^{23-25}$ It is worth mentioning that in some atypical luminescent materials, low-temperature phosphorescence or room temperature phosphorescence (RTP) and even ultra-long-lifetime RTP have been observed. ${ }^{23,24,59}$ Pure organic RTP materials have drawn considerable attention owing to their promising applications in varying fields, including biological imaging, molecular sensing, anti-counterfeiting and encrypted data storage. ${ }^{60-66}$ However, due to the spin inhibition nature of intersystem crossing (ISC) and the sensitivity of the triplet states to molecular motion, water, oxygen, etc., RTP of pure organic chromophores in solution is difficult to achieve. This also applies in the solid state: if the sample is hygroscopic or unable to effectively block oxygen, or the molecular conformation is not rigid enough, significant RTP cannot be observed.

Up to now, most reported RTP systems are chromophores with classic aromatic units and there are rare examples concerning non-conventional chromophores. ${ }^{59}$ Research on atypical luminescent chromophores has mainly focused on fluorescence, with little attention to phosphorescent emission. However, phosphorescence is widespread in atypical luminescent chromophores, which are more likely to produce triplet exciton states. The generation and stabilization of triplet states and their regulation are of great significance for phosphorescence. The following two factors have important influence on the generation and stabilization of triplet exciton states: First, if there are many double/triple bonds and/or heteroatoms, they can effectively enhance the spin-orbit coupling (SOC), thus promoting the ISC process and increasing the number of triplet excitons. Secondly, the clustering of an atypical chromophore could split energy levels and reduce the energy gap, which also facilitates ISC. Plus, hydrogen bonds could stabilize triplet states and enhance phosphorescence. Strong intramolecular and intermolecular interactions will form a 3D supramolecular network, which can effectively enhance the rigidity of the cluster chromophore. When the conformation is sufficiently rigid, triplet excitons should be stabilized, potentially resulting in ultra-long-lifetime RTP emission.
The above strategies for increasing the population of triplet excitons and/or stabilizing the triplet state to gain persistent phosphorescence directly coincide with the design principles of nonconventional chromophores. This specifically applies to introducing multi-hydrogen bonded networks and/or creating electron coupling interactions. Hydrogen bonds can trap excited electrons and suppress their non-radiative transitions. Moreover, chromophores without an aromatic structure and with long chain substituents prefer to form a coiled state, and the resulting intertwined structure can effectively isolate ambient oxygen and humidity, thus generating efficient pure organic RTP. In consideration of these points, atypical luminescent chromophores represent an ideal platform to realize effective RTP.

The following content summarizes design strategies of atypical PL/RTP chromophores. Revealing the relationship between the molecular structure, interactions and the luminescence in these chromophores will help to extend the scope of current purely organic PL/RTP systems and to further understand the origin of the emission. Additionally, the study of unconventional phosphorescent chromophores opens new opportunities to explore relationships between singlet states and triplet states, with the potential for utilization in optoelectronic sensors and display devices.

\section{Fluorescence systems}

Dendritic and hyperbranched poly(amido amine)s (PAMAM)s are the earliest and most widely studied polymeric materials containing atypical fluorescent chromophores. Initially, researchers found that PAMAM could emit weak fluorescence, ${ }^{67,68}$ then in 2003, Zheng et al. found strong blue fluorescence in the process of preparing gold nanoparticles using the fourth generation PAMAM (PAMAM-G4) as a template. However, due to the prepared gold nanoparticles also emitting strong blue fluorescence, the authors incorrectly attributed the PL of the composite system of PAMAM-G4 and gold nanoparticles to the PL of gold nanoparticles. ${ }^{69}$ Soon afterwards, Bard et al. first reported that dendritic PAMAM emitted strong blue fluorescence after oxidation..$^{52}$ However, Imae et al. soon overturned this oxidation mechanism. After adjusting the $\mathrm{pH}$ value, PAMAM dendritic chromophores with amino-hydroxy carboxylic acid at the end of each branch could emit strong blue fluorescence. Meanwhile, the fluorescence characteristics of PAMAM-G2 and PAMAM-G4 are quite different, and their intensity is directly related to the $\mathrm{pH}$ value. ${ }^{51}$ Three possible reasons have been speculated: (i) it may be that the protonated tertiary amine group fills the molecule with cations, and such strong Coulombic repulsion rigidifies the structure of PAMAM, so that the molecular chain segment movement is limited. PAMAM-G4, by contrast, is more crowded than PAMAM-G2 as the dendritic molecules grow outward and so PAMAM-G4 emitted with higher intensity. (ii) Hydrogen bond strength in PAMAM-G4 was increased under acid conditions, which could restrict and rigidity the movement of the molecules. (iii) A possible chemical reaction may occur between the lateral groups in PAMAM to produce a new fluorescent species. In 2007, Cao et al. reacted diethylene triamine with methyl acrylate at different 
ingredient ratios to obtain a series of $h b$-PAMAM derivatives. Their blue luminescence was also influenced by the $\mathrm{pH}$ value, the terminal groups $\left(\mathrm{NH}_{2}, \mathrm{NMe}_{2}\right.$ or $\left.\mathrm{CO}_{2} \mathrm{H}\right)$, the solvent, concentration and other factors. ${ }^{54}$ Since then, Imae et al. have further studied the PL mechanism of PAMAM. By comparing the fluorescence lifetimes and extent of protonation, they concluded that no new substance was formed after oxidation, and the oxidation of internal tertiary amine groups is the source of fluorescence. ${ }^{70}$

The more direct evidence that the amine groups of aliphatic amines are the active luminescent centers comes from the study of hyperbranched poly(ethylenediamine) ( $h b$-PEIs) and linear poly(ethylenediamine) (l-PEI) by Stiriba et al. in 2007. Two hyperbranched polymers and two linear polymers were compared, namely $h b$-PEIs, $l$-PEI and terminal methylated $h b$-PEIsMe and $l$-PEI-Me (Fig. 1). The fluorescence characteristics of these PEI derivatives are similar to those of previous PAMAM dendritic molecules: for example, oxidation and acidification can greatly enhance fluorescence. In addition, the fluorescence of PEI was enhanced after methylation. These results suggest that dendritic or hyperbranched structures are not necessary for fluorescence, but the presence of aliphatic amine groups is the key feature. ${ }^{53}$ In 2011, Yang et al. studied in detail the reaction between dendritic PAMAM and $\mathrm{H}_{2} \mathrm{O}_{2}$ by combining nuclear magnetic resonance (NMR) spectroscopy and MALDI-TOF mass spectrometry, which revealed the origin of PAMAM fluorescence was not intrinsic PAMAM but oxygenated PAMAM. When oxygenated with $\mathrm{H}_{2} \mathrm{O}_{2}$, PAMAM disintegrates through a cascade of Cope elimination reactions, resulting in fragments of hydroxylamine, which is the true fluorescent species (Fig. 2a). ${ }^{71}$

Although the PL mechanism of PAMAM has been controversial, work continues on improving the diverse fluorescence of PAMAM and exploring its biomedical applications. In 2009, Pan et al. reported a novel biodegradable $h b$-PAMAM (Fig. 2 b) ${ }^{72}$ They observed that the fluorescence intensity is molecular weight-dependent: increasing the molecular weight caused the chain segments to aggregate or crowd, inducing the fluorescence enhancement. $h b$-PAMAM can emit over a broad range of the visible spectrum depending on the excitation wavelength (Fig. 2c: $\lambda_{\text {ex }}$ 330-385, 460-490 and 510-550 nm gave blue, green and red

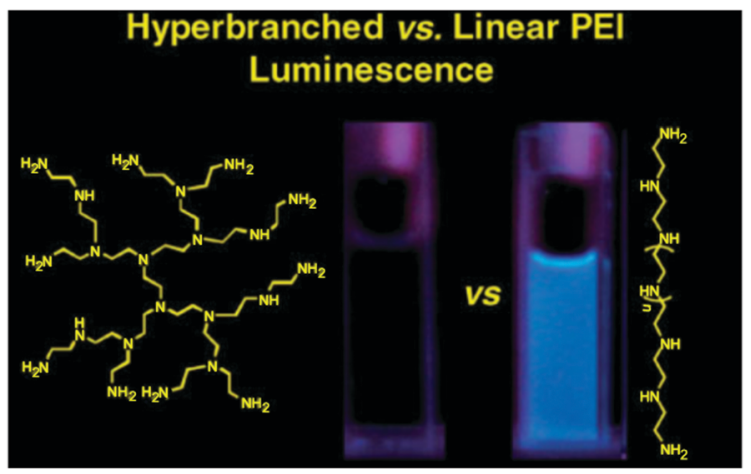

Fig. 1 Chemical structure and PL properties of the hyperbranched polyethylenimine and its linear counterpart. Reprinted with permission from ref. 53. Copyright 2007, John Wiley and Sons.

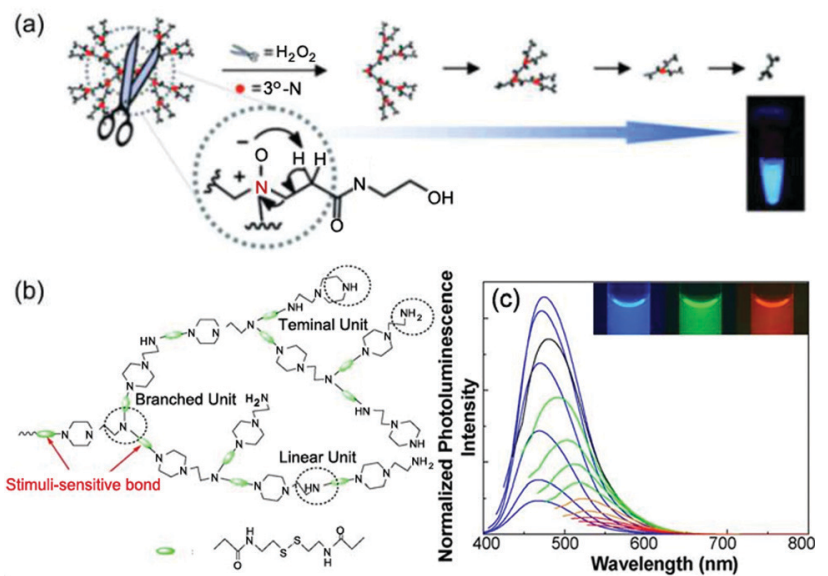

Fig. 2 (a) A plausible mechanism of PAMAM breakdown is proposed as a cascade of Cope elimination reactions. (b) An illustration of disulfidecontaining $h b$-PAMAM. Reprinted with permission from ref. 71. Copyright 2011, John Wiley and Sons. (c) Fluorescence spectra and images of $5 \mathrm{wt} \%$ aqueous solutions of the derived $h b$-PAMAM excited at different wavelengths. Reprinted with permission from ref. 72. Copyright 2009, John Wiley and Sons.

solutions, respectively). Later, they also found that peripheral modification of hyperbranched PAMAMs with mannose units could effectively limit the movement of chains, thus inhibiting the molecular collisions and self-quenching process, and thereby inducing significantly enhance fluorescence. ${ }^{73}$

According to above reports, it is apparent that (i) aggregation or steric crowding of chain segments can enhance fluorescence; (ii) the emission spectra show excitation dependence; (iii) limiting the movement of the ends of oligomer/polymer chains can further significantly enhance the luminescence. Up to this point, researchers had studied the molecular weight, topological structure, and $\mathrm{pH}$ of end-groups $\left(-\mathrm{OH},-\mathrm{NH}_{2}\right.$ and $\left.-\mathrm{COOH}\right)$, and concluded that these factors have important effects on the PL of PAMAM. However, these studies of systems containing aliphatic amines, such as PAMAMs and PEIs, were usually based on solutions with fixed concentrations, or in a narrow range of concentrations that are emissive. In this way, the PL behavior of polymers with different topological structures, and fractal dendrimers with different terminal groups at the same concentration, showed obvious differences.

In fact, the non-luminescent materials reported in the above literature can also produce effective emission after aggregation under appropriate conditions. In 2015, Zhu et al. revisited linear $(l)$ and hyperbranched $(h b)$ PAMAM (Fig. 3a). For hyperbranched PEI there was almost no emission from the dilute solution, but with increasing concentration, typical concentration-enhanced PL and AIE characteristics were observed (Fig. 3b). ${ }^{22}$ At certain concentrations, hyperbranched PEI shows weak emission, while linear PEI shows surprisingly stronger emission. Experiments have shown that the difference between the hyperbranched and linear PEI solution emission mentioned above are essentially caused by the aggregation state of $\mathrm{N}$ atoms and the level of conformational hardness. Thus, topological structure is not the determinant of PAMAM luminescence, and the proposal that 
(a)
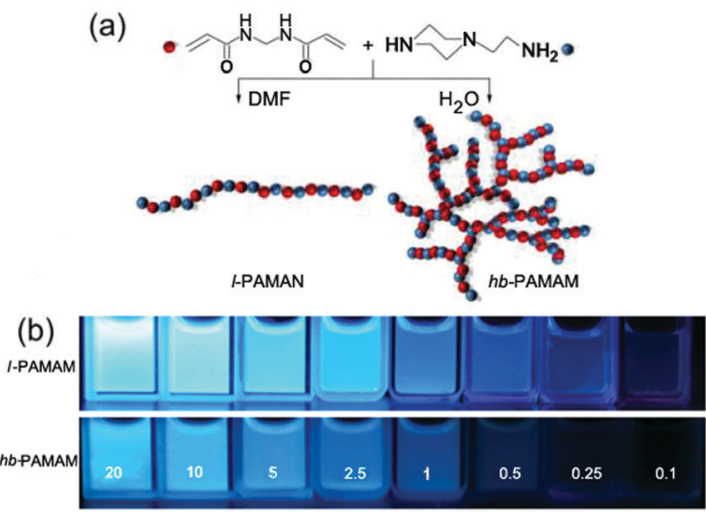

Fig. 3 (a) Synthetic route to linear $(l)$ and hyperbranched $(h b)$ PAMAMs (b) Photographs taken under UV irradiation of aqueous solutions of l-PAMAM and $h b$-PAMAM at different concentrations. Reprinted with permission from ref. 22. Copyright 2015, Springer.

oxidation is the root of PAMAM luminescence was also excluded, while the AIE mechanism underlying the luminescence has been broadly recognized. More importantly, this study extended AIE systems from traditional conjugated chromophores to unorthodox luminous chromophores. Due to the combination between excellent biocompatibility of unorthodox chromophores and strong solid-state fluorescence of AIE systems, these unorthodox chromophores have become promising candidates for bioimaging, such as bacterial detection, which expands the bioapplications of AIE systems, and stimulates the exploration of more novel unconventional luminogens.

Actually, the AIE phenomenon was perceived in early studies. In 2007, Tang et al. reported AIE of a maleic anhydride-alt-vinyl acetate copolymer (PMV). Its THF solution is very weakly emissive, but its nanometer suspension in butyl acetate emits bright blue light. ${ }^{56}$ Although this remarkable behavior of PMV could not be reasonably explained at that time, subsequent work has attributed the PL of PMV to the clustering of carbonyl groups in a locked state, which becomes an effective model for the study of atypical luminescent materials. ${ }^{74-76}$

Subsequently Pucci et al. reported polyisobutene succinic anhydrides PIBSA (a) and PIBSA (b), and their succinimide (PIBSI) counterparts with AIE characteristics, which were attributed to the aggregation of carbonyl groups which in turn restricted the vibrational motions and reduced nonradiative relaxation (Fig. 4). They also demonstrated that the AIE is strongly dependent on the number and nature of interacting chromophores grafted onto the polymeric backbone. The reasonable explanation is that more subgroups facilitate the formation of more clusters, and the enhanced interaction intensity further solidifies the clusters thus providing enhanced emissions. ${ }^{77}$

In 2013, Yuan et al. found that rice, starch and fibronectin not only emit fluorescence under ultraviolet light, but also emit room temperature phosphorescence (RTP) in the solid state. ${ }^{58}$ They hypothesized that the large number of oxygen atoms in the structure was the source of the luminescence. Clearly the isolated oxygen atoms could not be excited by ultraviolet light and would not emit visible light, thus the authors proposed
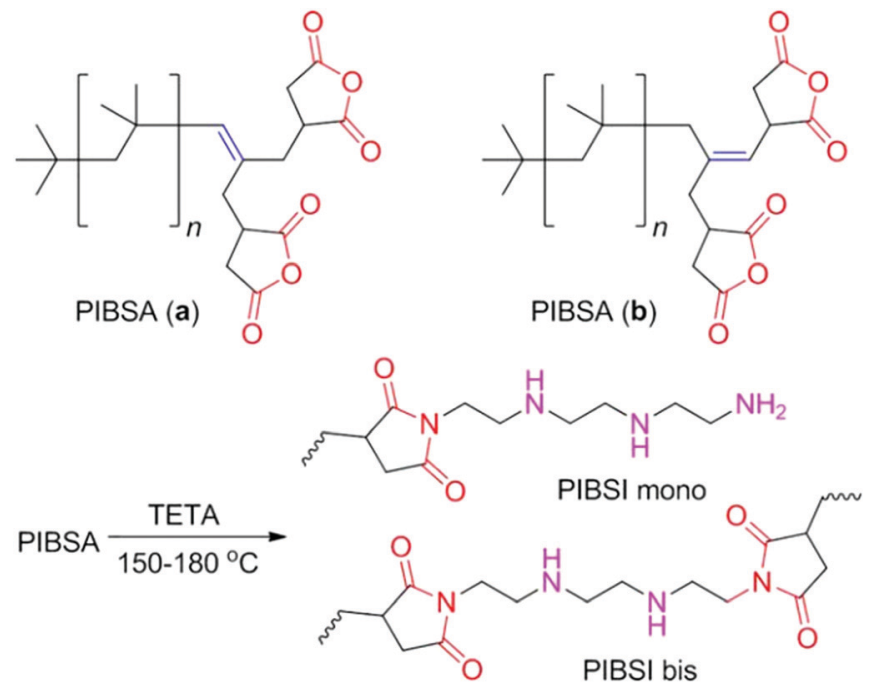

Fig. 4 Bis-succinic anhydride derivatives PIBSA (a) and PIBSA (b), and their triethylenetetramine (TETA) decorated corresponding mono and bisimides (PIBSI mono, PIBSI bis). Reprinted with permission from ref. 77. Copyright 2008, John Wiley and Sons.

that clusters of oxygen atoms gave the luminescence to these natural products. Clustered oxygen atoms can easily promote electron interactions and delocalization. The clusters increase the effective conjugation length and harden the conformation so that these regions are excited by UV light. This phenomenon was later named cluster-triggered emission (CTE) and was identified as a new category of AIE property. In addition, there are many hydrogen bonds in these systems, which not only makes the conformation rigid, but also makes it easier for oxygen atoms to contact each other at a short distance, thus enhancing the luminescence. At the same time, the entangled dispersive interactions of the polymer chains are beneficial to the conformational hardening of the cluster chromophore, reducing the non-radiative transition and improving the luminous efficiency.

The introduction of the concept of CTE provided a deeper understanding of the underlying mechanisms of emission in early atypical luminescent chromophores. For instance, in the above PIBSA (a) and PIBSA (b), in addition to carbonyl groups, virtually all succinic anhydride (SA) and succinimides (SI) groups, as well as amine groups, may be involved in the emission (Fig. 4). When these units aggregate in a concentrated solution or film, the overlap of the electron clouds leads to the extension of conjugation and forms a hardened conformation, resulting in emission. In 2016, Li et al. uncovered and studied the unconventional PL of sulfonated acetone formaldehyde (SAF), acetone formaldehyde (AF) condensates and sulfonated ethylenediamineacetone-formaldehyde (SEAF) and sulfonated phenol-acetoneformaldehyde (SPAF) (Fig. 5). ${ }^{38,78}$ The authors ascribed the emission mechanism of these polymers to the cluster of carbonyl groups which was induced by strong ionic and hydrogen bonding interactions. However, the clustering and electronic communication of phenol, hydroxyl, amine and sulfonyl groups may also contribute to the fluorescence enhancement. ${ }^{79}$ 
(a)
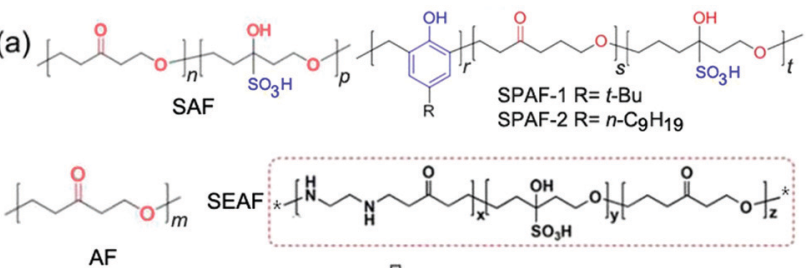

AF

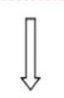

(b)

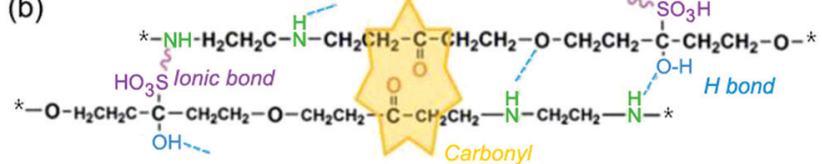

Fig. 5 (a) Chemical structures of AF, SAF, SEAF, and SPAF. (b) A schematic diagram of a carbonyl cluster, ionic and hydrogen bonds among SEAF chains. Reprinted with permission from ref. 38 and 78. Copyright 2016, Royal Society of Chemistry.

The enhanced understanding of the CTE mechanism also helps the discovery and design of new atypical materials. Based on the CTE mechanism, Yuan et al. designed and synthesized a series of polyurethane (PU), ${ }^{45}$ polysulfide (P1), polysulfoxide (P2) and polysulfone (P3) derivatives (Fig. 6). ${ }^{37}$ Clustering of NHCOO- in PUs, and clustering of COO- and sulfur-containing units $(\mathrm{S}, \mathrm{S}=\mathrm{O}, \mathrm{O}=\mathrm{S}=\mathrm{O}$ ) activates emission from these polymers. It is worth mentioning that the sequential oxidation from P1 to P3 gradually improves the luminous efficiency, showing a certain degree of tunability.

The tuning of absorption and PL spectra is also very important. In 2015, Li et al. reported that a polythioamide derivative (PTAM) showed different colors in dilute solution and solid states (Fig. 7). PTAM solids exhibit remarkable red-shifted yellow fluorescence, consistent with tighter electron interactions and a more rigid conformation in solid than in solution. Meanwhile, H-bonds and

(a)
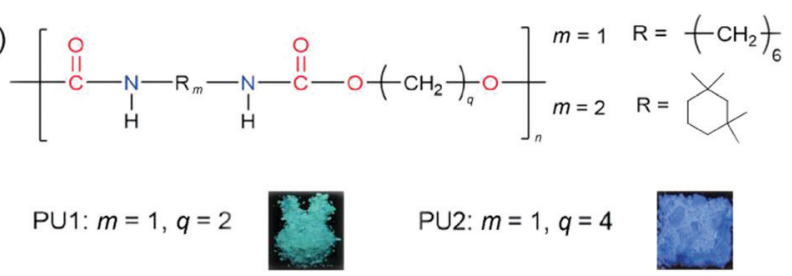

PU3: $m=2, q=2$

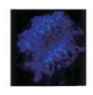

PU4: $m=2, q=4$

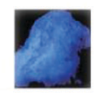

(b)

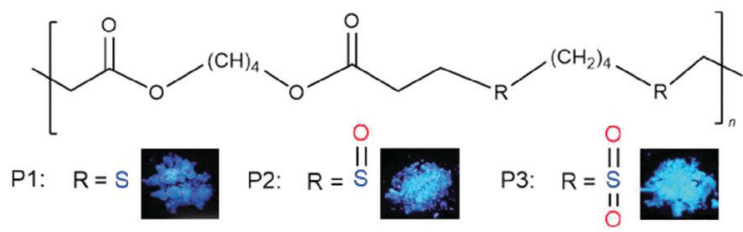

Fig. 6 Structure and photographs of solid powders taken under UV light for (a) PU1-PU4 and (b) P1-P3. Reprinted with permission from ref. 45 and 37. Copyright 2019, Royal Society of Chemistry.

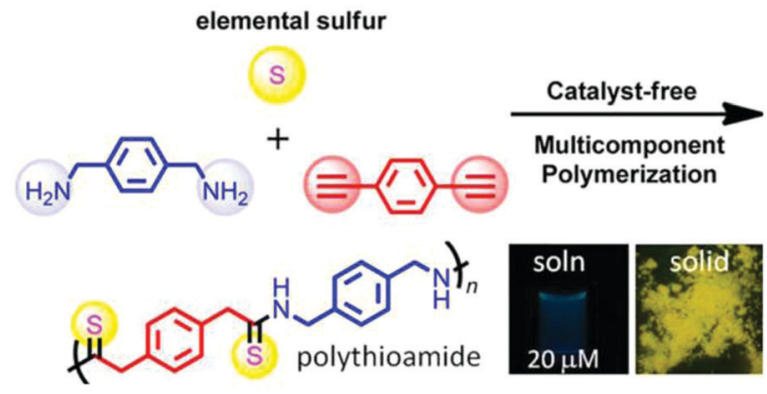

Fig. 7 Multicomponent polymerization for PTAM synthesis. The insets are the photographs of DMF solution and solids of PTAM taken under $365 \mathrm{~nm}$ UV light. Reprinted with permission from ref. 35. Copyright 2015, American Chemical Society.

$\mathrm{n}-\pi$ interactions between $\mathrm{C}=\mathrm{S}$ and $-\mathrm{NH}$ groups, facilitate heteroatom clusters. Additionally, $n-\pi$ interactions (between phenyls and heteroatoms) and $\pi-\pi$ interactions contribute to the fluorescence of PTAMs. $^{35}$

In 2015, Tang et al. thoroughly studied the solvatochromic properties of PMV (Fig. 8). ${ }^{76}$ After interaction with an electronrich solvent, its absorption and emission are red shifted due to clustering groups complexing with the solvent, without changing the chromophore. Notably, magenta emission $\left(\lambda_{\max } 619 \mathrm{~nm}\right)$ was obtained in DMF solution although PMV has no traditional $\pi$-conjugated system or any phenyl rings. Such a system should have excellent biocompatibility and biodegradability. Therefore, this development provides a good molecular design idea for future applications of atypical luminescent materials in biology.

The above PMV, however, does have carbonyl bond chromophores in its structure. Li et al. reported a fluorescent aliphatic hyperbranched polyether epoxy (EHBPE). ${ }^{48}$ There are no carbonyl units or aryl rings in EHBPE, and oxygen atoms are present as ether and hydroxyl groups. Therefore, the strong fluorescence observed was unexpected. Under an ultraviolet lamp, EHBPE in the bulk state shows obvious strong blue-green fluorescence (Fig. 9b). In this state intramolecular and intermolecular interactions block the free rotation of $\mathrm{C}-\mathrm{C}$ and $\mathrm{C}-\mathrm{O}$ bonds of the polymer chains, which is very similar to the restricted intramolecular rotation

(a)

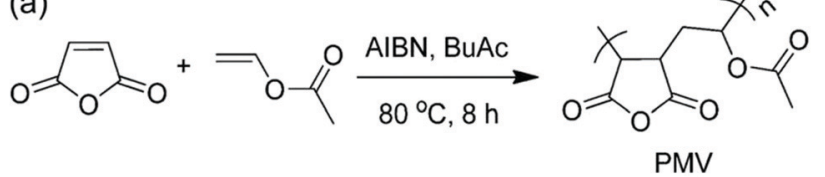

(b)

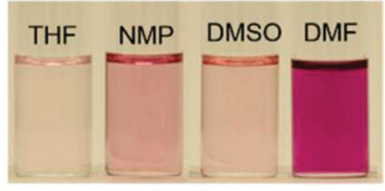

Daylight

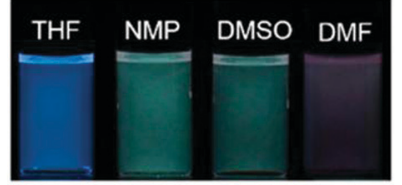

UV
Fig. 8 (a) Synthetic Route to PMV. (b) Photographs of PMV in different solvents were taken under (left) daylight and (right) $365 \mathrm{~nm}$ UV light illumination. Reprinted with permission from ref. 76. Copyright 2015, American Chemical Society. 
(a)

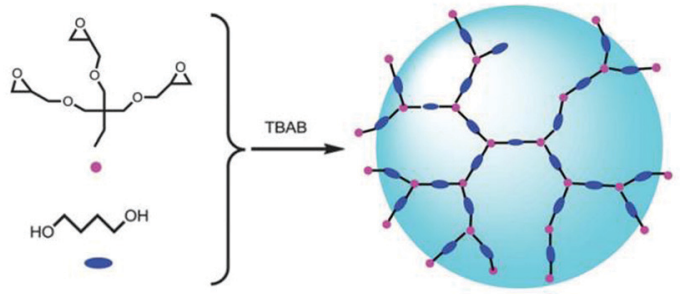

(b)
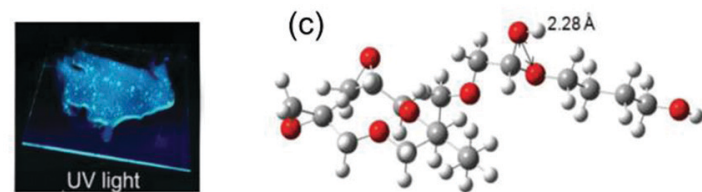

Fig. 9 (a) Schematic representation of the synthetic route for EHBPE. (b) Images of EHBPE in bulk under UV irradiation. (c) Optimized conformation of P1. Reprinted with permission from ref. 48. Copyright 2016, Royal Society of Chemistry.

(RIR) process in AIE systems. RIR blocks the non-radiation pathways and opens radiative channels in the aggregated state. In order to better understand the photophysical processes, the conformation of P1 (an oligomer model of EHBPE) was simulated. The simulation shows that there may be interaction between ether and hydroxyl oxygen atoms due to their close proximity (Fig. 9c). Dense clusters of oxygen (ether and hydroxyl oxygen) could enhance interactions and (a)

(b)

(d)

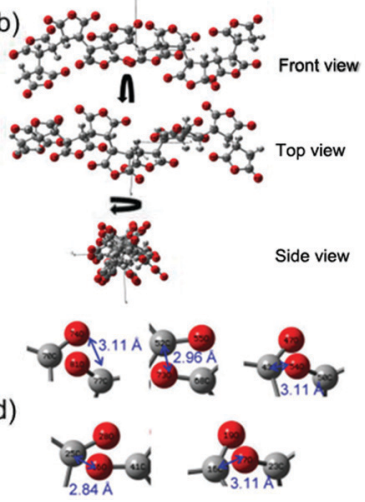

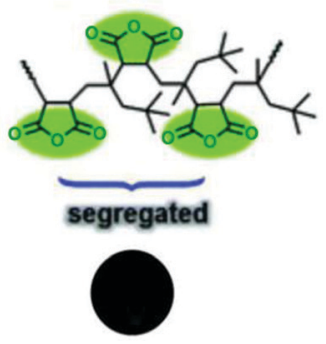

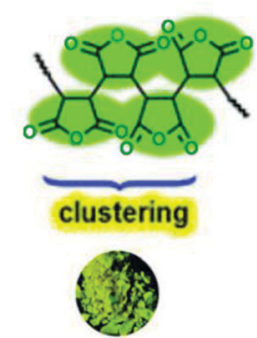

(c)

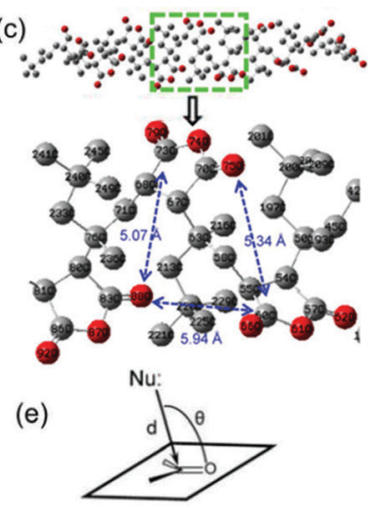

Fig. 10 (a) Chemical structures of PMP and OMAh4 and photos of segregated and clustering states. (b) Optimized conformation of OMAh4 at different views. To simplify the calculation, ten repeating units of both OMAh4 and PMP were used. (c) Optimized conformation of PMP (hydrogens on anhydride rings were omitted for the concise view). (d) The interaction types of carbonyl groups in OMAh4. (e) The proposed model of $n-\pi^{\star}$ interaction. To simplify the calculation, ten repeating units of both OMAh4 and PMP were used. Reprinted with permission from ref. 80. Copyright 2017, Royal Society of Chemistry. produce emission. Therefore, the high density oxygen clusters in and between molecules may be AIE active, and the emission of the high density clusters of heterogeneous clusters may be related to the lone pair electrons, which promote the abnormal fluorescence.

Much research is currently focused on trying to get a more accurate description of the mechanism of cluster luminescence at the atomic level. In 2017, Tang et al. reported poly[(maleic anhydride)-alt-(2,4,4-trimethyl-1-pentene)] (PMP) and oligo(maleic anhydride)s (OMAh4) with clusteroluminescence. They employed density functional theory to optimize the conformations of OMAh4 and PMP. Theoretical simulation showed that the distance between two adjacent MAh units of OMAh4 was 2.84 ̊-3.18 A; however, for PMP, the comparable distance was much longer, $4.90 \AA-5.37 \AA$ A. Thus, OMAh4 could generate intra- and inter-chain $n-\pi *$ interactions of the carbonyl groups, resulting in the luminescent rigidified clusters (Fig. 10). ${ }^{80}$

In 2018, Yuan et al. reported crystallization-enhanced emission of poly(ethylene terephthalate) (PET), which also shows concentration-enhanced and AIE properties (Fig. 11). PET is weakly luminescent in dilute solutions, while efficiently luminescent in concentrated solutions or films. Additionally, PET exhibits only fluorescence in solutions, while it generates fluorescence/phosphorescence dual emission in the solid state at room temperature. Results showed that conformational rigidification enhances the luminous efficiency of PET films, by crystallization. Such crystallization enhanced dual emission could be explained by the CTE mechanism. Efficient throughspace electronic conjugation of clusters of terephthalate units leads to extended delocalization. ${ }^{81}$

In 2019, Yan et al. designed and synthesized a series of polysiloxane (HPS) derivatives without a typical luminous unit, but all showed intrinsic emission, with applications in biological fields. Fig. 12 shows the structure of HPS. Its PL is attributed to the $\mathrm{Si} \cdot \mathrm{O}$ interactions, and $n-\pi^{*}, \pi-\pi$ interactions between the oxygen atom $(=\mathrm{O}-)$ and $\mathrm{C}=\mathrm{C}$ bonds. Such intrinsic $\mathrm{PL}$ and concentration enhanced PL could be reasonably explained by CTE. ${ }^{82}$

Initially, compared with traditional conjugated luminescent materials, the reported atypical luminescent materials generally

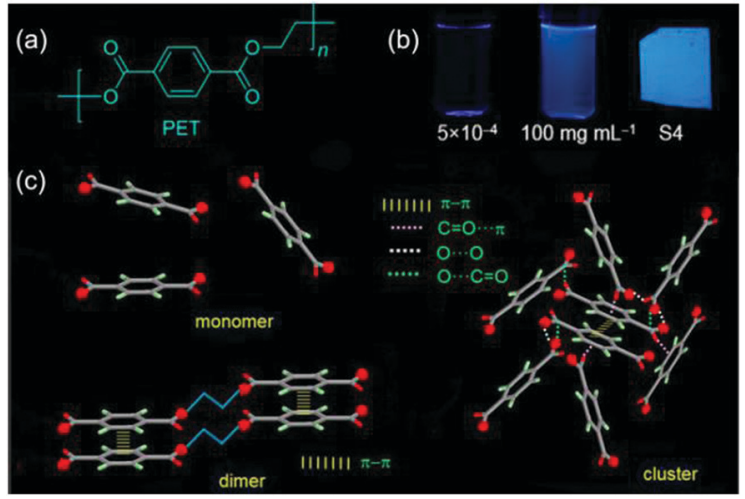

Fig. 11 (a) Chemical structures of PET. (b) Dilute and concentrated solution and film of PET. (c) Schematic illustration of monomer, dimer, and cluster in PET solids. Reprinted with permission from ref. 81. Copyright 2018, American Chemical Society. 


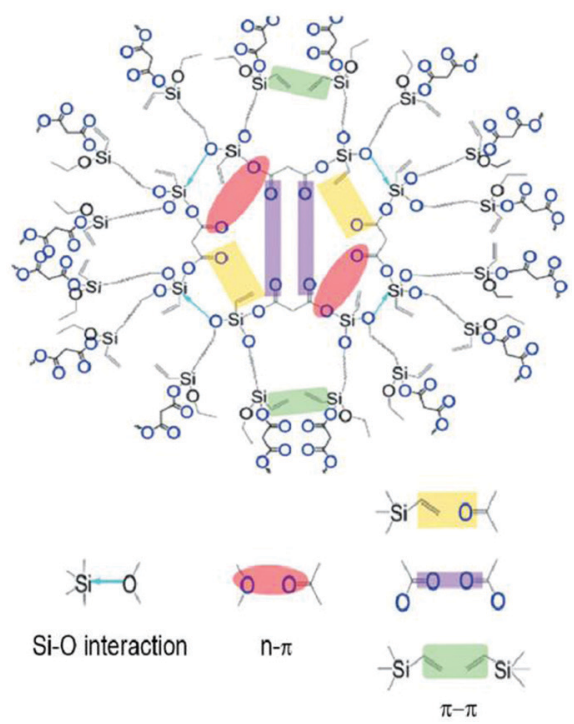

Fig. 12 Structure and schematic diagram of electronic interactions among different groups of HPS. Reprinted with permission from ref. 82 Copyright 2019, American Chemical Society.

had lower fluorescence quantum yields (PLQYs) and their PL was usually in the blue light region. Although some polymers could glow red, it is difficult to guarantee high PLQYs, limiting their use in biological field. Generally, the longer excitation and emission wavelength is less harmful to cells and tissues and can penetrate more easily through the thicker samples. More flexible chain structures can adopt more conformations, which is beneficial for intra- and/or inter chain interactions, influencing the aggregation structures and hence producing red-shifted emissions. There are some ways to increase the flexibility of molecular chains. For the main chains, the existence of double bond should benefit flexibility, because of the limited steric hindrance. For side chain substituents, high symmetry can lead to good flexibility. Also, the molecular weight is another factor that affects the formation of appropriate conformations. A longer chain would bring more conformations and be more likely to fold or curl, thus giving better flexibility. Therefore, to achieve efficient and red-shifted emission appropriate chromophores should be introduced as well as controlling the physical structures of the molecular chains.

Progress was soon made in this area. In 2017 Wang et al. synthesized alternating copolymers PMVP2 and PMVP10 of $N$-vinyl pyrrolidone and maleic anhydride with different molecular weights (Fig. 13a). For PMVP2 with high molecular weight, the strong entanglement of its long chains inhibited the aggregation of anhydride and amide groups, while PMVP10 with low molecular weight is more prone to aggregation, and could form strong intra-chain and/or inter-chain $n-\pi^{*}$ and $\pi-\pi^{*}$ interactions, so as to regulate the clustering state and tune the luminous color. ${ }^{83}$

Interestingly, poly(itaconic anhydride-co-vinyl pyrrolidone) (PIVP) with a lower chain rigidity shows unusually strong red fluorescence at excitation of about $600 \mathrm{~nm}$ (Fig. 13c), which sheds some light on preparing red-shifted nonconventional (a)
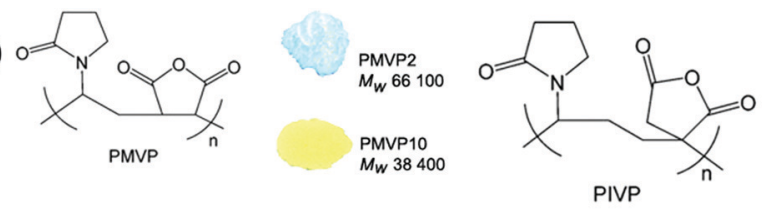

(b)
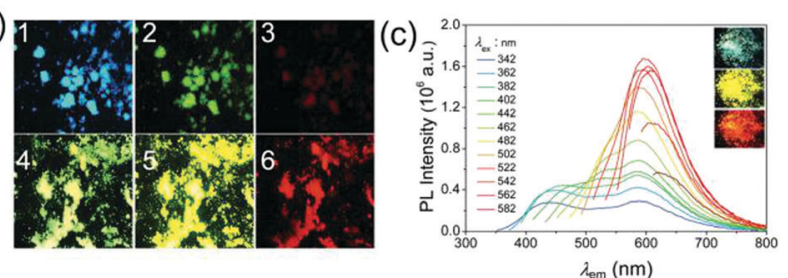

Fig. 13 (a) Structure of PMVP and PIVPs, and photographs taken under $365 \mathrm{~nm}$ UV light of solid powders for PMVP with different molecular weights. (b) Fluorescence microscope photographs of PMVP-2 (1-3) and PMVP-10 (4-6) powders under light irradiation at different excitation wavelengths. From left to right: UV light $(360-375 \mathrm{~nm})$, blue light $(470-495 \mathrm{~nm})$, and green light $(540-550 \mathrm{~nm})$, respectively. (c) PL spectra and photographs of PIVP-10 powder at different excitation wavelengths. Reprinted with permission from ref. 83. Copyright 2017, Royal Society of Chemistry.

luminescent polymeric materials. ${ }^{83}$ The emission of PMVP could cover the whole visible spectra depending on molecular weight and excitation wavelength. In 2019, Qiao et al. mixed solid and $\mathrm{NaOH}$ solution with different molar ratios to obtain a solid with luminescent color ranging from cyan to red (Fig. 14). ${ }^{84}$ Obviously, the addition of $\mathrm{NaOH}$ changed the clustering state of the chromophore. This mechanism has not been fully elucidated, but it demonstrates the possibility of regulating the optical properties of atypical luminescent chromophores. Next, Yan et al. reported the hyperbranched poly(amino ester)s (PAE)s with multicolor photoluminescence. PAE's distinct AIE character derived from the clustering of ester and tertiary amine groups. Moreover, PAE exhibits tunable fluorescence from blue, cyan and green to red by adjusting the excitation wavelength (Fig. 15). ${ }^{85}$ Zhang et al. reported that poly(4-(cyanomethyl)phenyl metha) (PCPMA) showed a hyperbranching-enhanced-emission (HEE) effect. Cluster chromophores formed via through-space conjugation (TSC), inducing the HEE effect, which strongly promoted PL (Fig. 16). ${ }^{86}$ The HEE effect provides a universal strategy to expand the current range of highly efficient atypical chromophores.

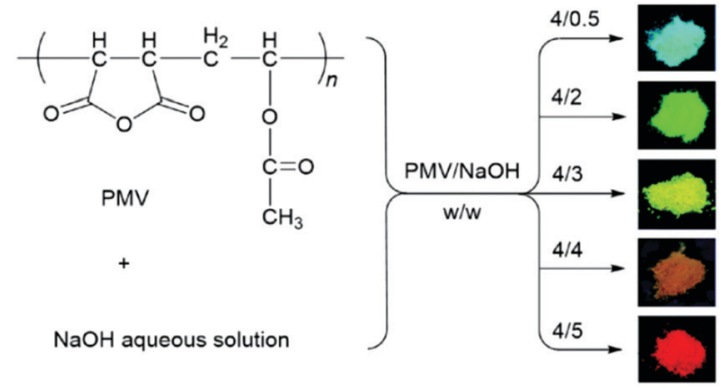

Fig. 14 Preparation strategy of new photoluminescent polymers and photographs of their emission. Reprinted with permission from ref. 84 . Copyright 2019, Royal Society of Chemistry. 


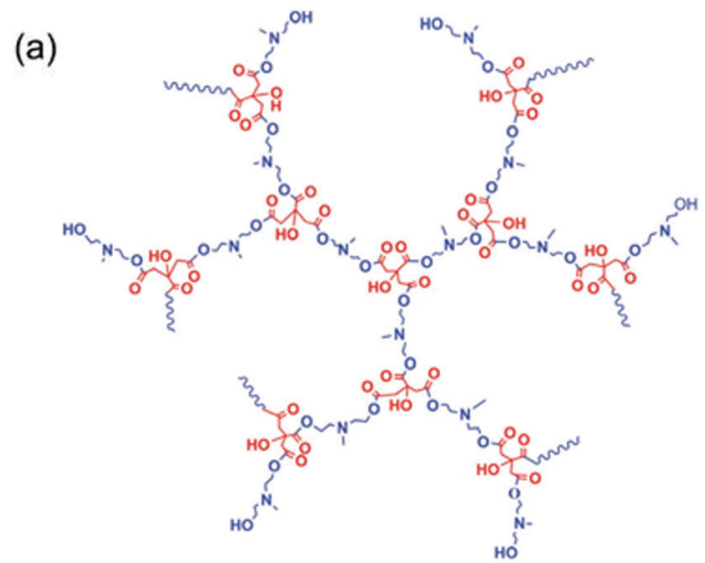

(b)

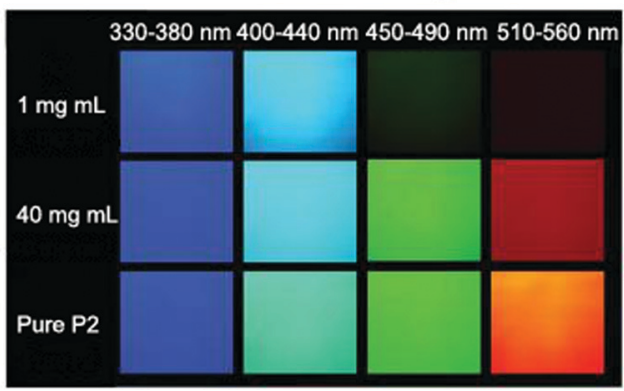

Fig. 15 (a) The structure of poly(amino ester)s. (b) Fluorescence microscope images of P2 solution and pure P2 under the light filter of UV-2A (Ex 330-380 nm, DM 400, BA 420); BV-2A (Ex 400-440 nm, DM 455, BA 470). Reprinted with permission from ref. 85. Copyright 2019, John Wiley and Sons

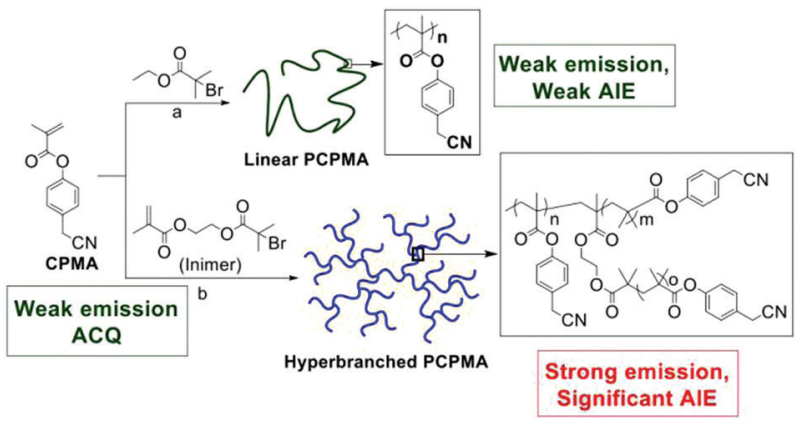

Fig. 16 Synthesis of linear and hyperbranched PCPMA. Reprinted with permission from ref. 86. Copyright 2019, American Chemical Society.

Up to now, the AIE mechanism is still guiding the synthesis of atypical luminescent chromophores. Electron-rich heteroatoms, such as nitrogen, oxygen, phosphorus and sulfur, and/or unsaturated $\mathrm{C}=\mathrm{C}$ and $\mathrm{C}=\mathrm{N}$ bonds, have been reported frequently to act as unconventional luminescent subunits when aggregated. In 2020, De et al. utilized hydrated polymer PNVCL (Fig. 17), without any classical fluorophore entity, as a novel fluorescent thermometer for intracellular temperature determination. When the temperature increased to above its lower critical solution temperature, $38{ }^{\circ} \mathrm{C}$, which is near to human body temperature, the conformation changed from coil to globular and fluorescence was greatly enhanced (Fig. 17). ${ }^{28}$ This work further (a) Non-fluorescent $\longrightarrow$ Weakly fluorescent $\longrightarrow$ Highly fluorescent

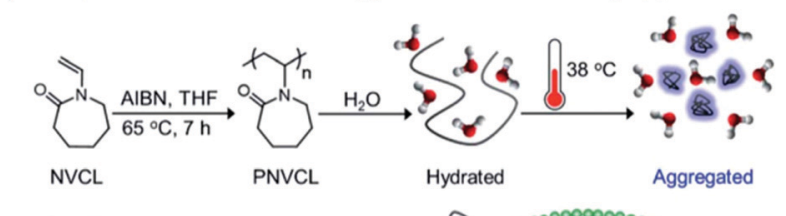

(b)

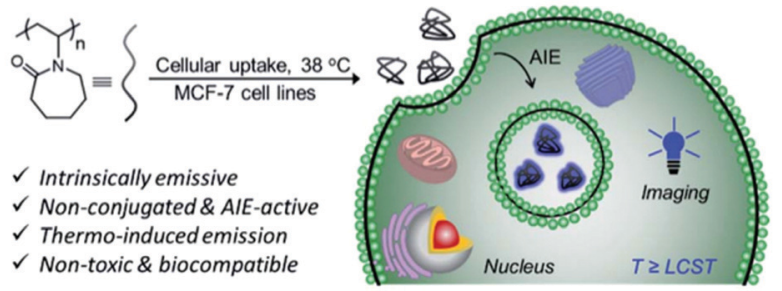

Fig. 17 (a) Synthesis of PNVCL via free radical polymerization and its thermally-induced conformational transformation. (b) Overall schematic illustration of intracellular temperature imaging using non-conjugated PNVCL as a fluorescent thermometer. Reprinted with permission from ref. 28. Copyright 2020, Royal Society of Chemistry.

demonstrates that the fluorescence of an unconventional system in the aggregated state originates not from a specific entity with a definite structure, but plausibly from interaction among chromophores with heterogeneity and complexity.

In 2020, Wang et al. reported a fluorous effect-induced emission of azido substituted poly(vinylidene fluoride) with high photostability and film formation, which displays a strong excitation-dependent emission behaviour in both solution and solid-state (Fig. 18). In this work, azido groups serve as a new kind of unconventional chromophore and the microfluorous environment leads to a stable excited state and high fluorescence efficiency by decreasing the energy of the HOMO and LUMO. The combination of fluorine substituents with an atypical chromophore was shown to produce good solution processability, excellent mechanical flexibility, high photostability and high PLQYs. ${ }^{87}$ The stimulation of clustered chromophores by external forces could change the distance and relative position between the individual subunits. This would affect intermolecular interactions and electron delocalization, thereby adjusting the polymers' luminescent properties. This process gives aliphatic polymers largely unexplored potential applications in the field of mechanoresponsive materials.

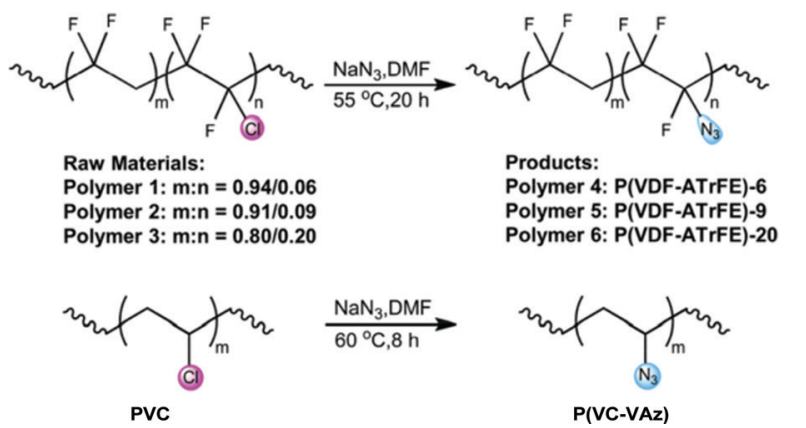

Fig. 18 Synthesis strategy of P(VDF-ATrFE) unconventional fluorescent polymers and azido substituted PVC (P(VC-VAz)). Reprinted with permission from ref. 87. Copyright 2020, Royal Society of Chemistry. 


\section{RTP systems}

As mentioned above, low-temperature phosphorescence or RTP and even ultra-long-lifetime RTP have recently been widely observed in atypical luminescent materials. For instance, in 2016, Yuan et al. reported CTE of polyacrylonitrile. Indeed, as shown in Fig. 19a and b, when the ultraviolet lamp was turned off, significant ultra-long lifetime green phosphorescence was observed at $77 \mathrm{~K}$ because the triplet excitons were stabilized by the low temperature. More importantly, PAN solids exhibit RTP (Fig. 19c), although the phosphorescence lifetime is short $(0.2 \mathrm{~ms})$. The unique emission of PAN is ascribed to the formation of "cyano clusters" as the chromophores. In such clusters, $\pi$ and lone pair (n) electrons among cyano groups overlap to extend the conjugation and simultaneously rigidify the conformation, thus offering remarkable emission. The presence of lone pairs promoted ISC transitions and triplet emission of PAN powders, leading to RTP. ${ }^{39}$ In 2018, Su et al. synthesized a series of nonconjugated polyurethane derivatives. Long-persistent phosphorescence is observed at $77 \mathrm{~K}$. The mechanism for this unusual behavior has been shown by steady-state photophysical characterization and time-resolved emission spectra to arise from the formation of intra and/or intermolecular carbonyl clusters at low temperature (Fig. 20). The lifetime of long-persistent phosphorescence is increased by the introduction of an aromatic monomer into the nonconjugated polyurethane chains. This is attributed to intra- and/or intermolecular $n-\pi^{*}$ transitions from electron-rich carbonyl groups to the conjugated aromatic units, thereby enhancing the ISC rate. ${ }^{88}$

In 2018, Yuan et al. revealed the phosphorescence of PEG and $\mathrm{F} 127$ at $77 \mathrm{~K}$ and ultra-long phosphorescence of xylitol at room temperature, as shown in Fig. 21. There are no effective hydrogen bond interactions in PEG and F127 solid, whereas xylitol could form hydrogen bonds which are conducive to the stability of triplet excitons, which could explain the ultra-long RTP of xylitol. ${ }^{47}$

It is worth noting that the generation of triplet excitons of atypical luminescent chromophores does not depend on the existence of hydrogen bonds, as proved by the RTP of PAN and ultra-long lifetime phosphorescent properties of PAN and PUs from above. However, the results with PEG and F127 showed that although the presence or absence of hydrogen bonds is not

(b)

(a)

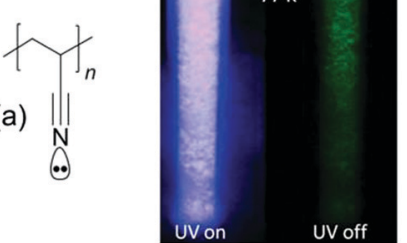

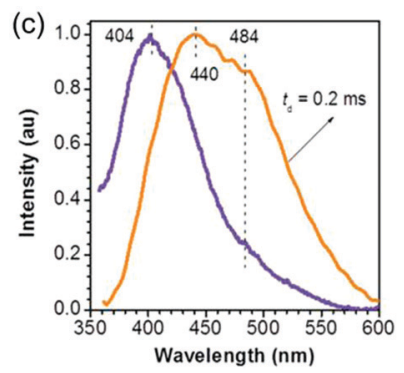

Fig. 19 (a) Structure of PAN. (b) Photographs of PAN powders taken at $77 \mathrm{~K}$ under $365 \mathrm{~nm}$ UV light or after ceasing the UV irradiation. (c) Emission spectra of PAN powders with $t_{\mathrm{d}}$ of 0 and $0.2 \mathrm{~ms}\left(\lambda_{\mathrm{ex}}=335 \mathrm{~nm}\right)$. Reprinted with permission from ref. 39. Copyright 2016, John Wiley and Sons.

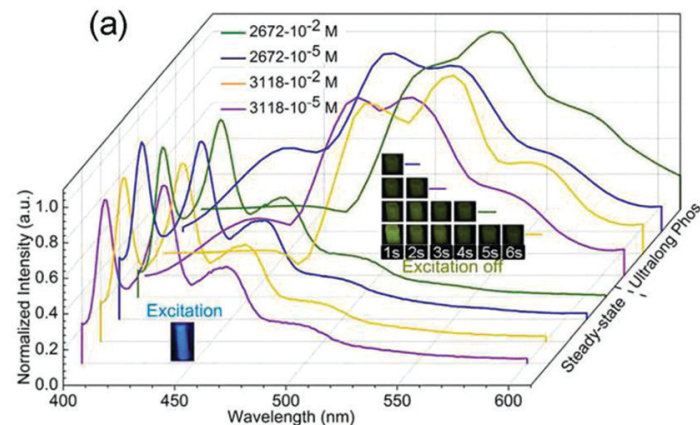

(b)

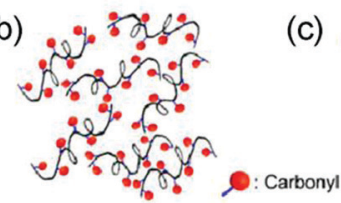

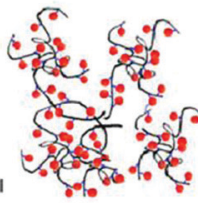

(d)

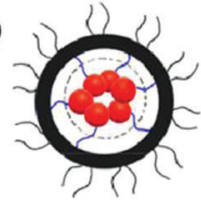

Fig. 20 (a) Steady-state PL and long-phosphorescence spectra of PU1 with different average molecular weights $\left(M_{n}=2672\right.$ or $\left.3118 \mathrm{~g} \mathrm{~mol}^{-1}\right)$ and different concentrations in 2-MeTHF solutions at $77 \mathrm{~K}$. And schematic illustration of PU1 (b) dilute solution, (c) concentrated solution, and (d) carbonyl clusters in the aggregated state. Reprinted with permission from ref. 88. Copyright 2018, American Chemical Society.

(a)

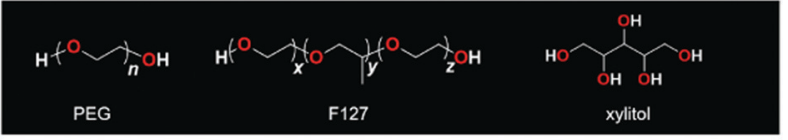

(b)

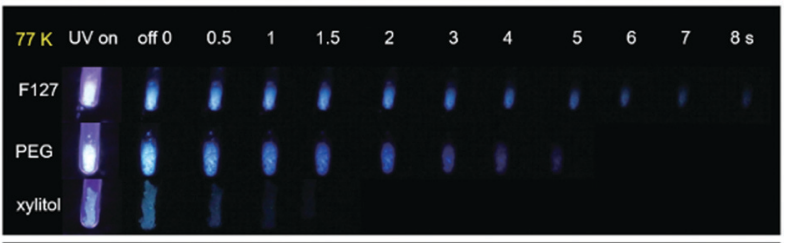

(c)

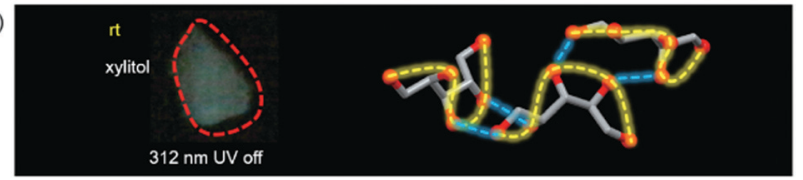

Fig. 21 (a) Structures of PEG, F127, and xylitol. (b) Photographs of the solid powders of F127, PEG, and xylitol taken under $312 \mathrm{~nm}$ UV light or after ceasing the UV irradiation at $77 \mathrm{~K}$. (c) Photograph of xylitol powders taken at room temperature after ceasing the $312 \mathrm{~nm}$ UV irradiation, and partial electronic channels of $\mathrm{O}$... O short contacts in xylitol crystals. Reprinted with permission from ref. 47. Copyright 2018, John Wiley and Sons.

the key to phosphorescence, the presence of hydrogen bonds could promote conformational hardening, thus limiting nonradiative transitions and ultimately facilitating the generation of RTP. In addition, xylitol single crystal data showed that effective intramolecular/intermolecular interactions were formed between $\mathrm{O}$ atoms (Fig. 21c), which once again proved the rationality of the CTE mechanism. ${ }^{47}$

Also in 2018, Zhang et al. observed that poly-L-lysine ( $\varepsilon$-PLL) powder emits blue-white light under $365 \mathrm{~nm}$ ultraviolet irradiation, and green ultra-long RTP could be seen when the light is turned off. The spectrum shows that the instantaneous emission has a typical excitation wavelength dependence, and the RTP is also different, 
(a)

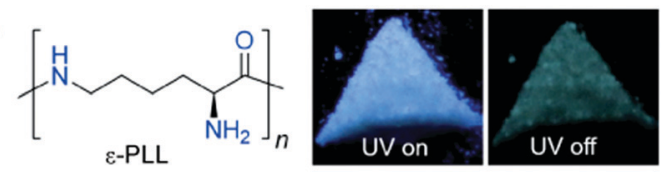

(b)

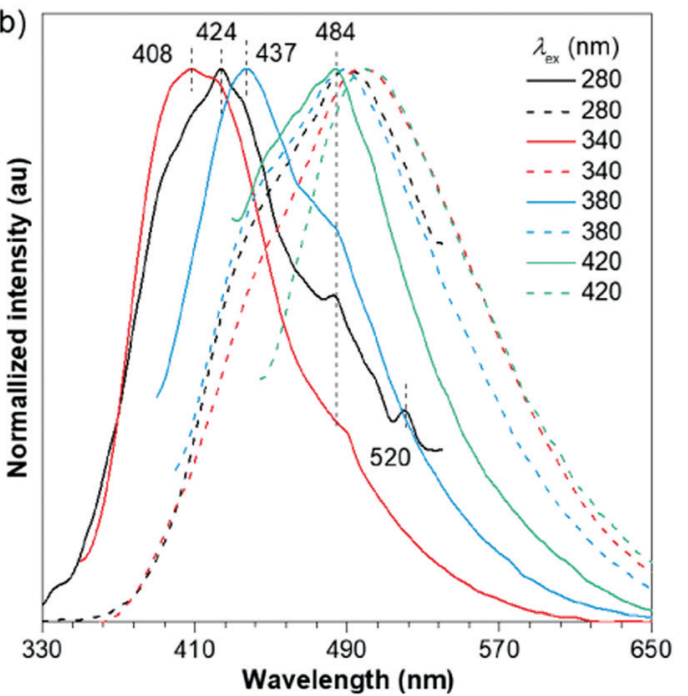

Fig. 22 (a) Structure of $\varepsilon$-PLL and the photograph of its powders taken under $365 \mathrm{~nm}$ UV light or after ceasing the irradiation. (b) Normalized emission spectra of $\varepsilon$-PLL powders with $t_{d}$ of 0 (solid line) and $0.1 \mathrm{~ms}$ (dash line) under varying $\lambda_{\text {ex }}$. Reprinted with permission from ref. 89. Copyright 2018, Springer

with a peak from 489 to $500 \mathrm{~nm}$ (Fig. 22). Clustering of nonconventional chromophores, which ensures subsequent electron cloud overlap, together with conformation rigidification is responsible for the emission. Single crystal analysis reveals the prevalence of such through-space electronic communications as $\mathrm{O} \cdots \mathrm{O}$, $\mathrm{C}=\mathrm{O} \cdots \mathrm{N}, \mathrm{O}=\mathrm{C} \cdots \mathrm{C}=\mathrm{O}$, and $\mathrm{O}=\mathrm{C} \cdots \mathrm{O}=\mathrm{C}$ short contacts, which construct interpenetrated 3D networks. ${ }^{89}$ In 2019, Yuan et al. proved that the PL of three common polymers: polyacrylic acid (PAA), polyacrylamide (PAM) and poly( $n$-isopropyl acrylamide) (PNIPAM) was intrinsic rather than due to the impurity PL or through the PL of their monomers. ${ }^{90}$ The clustering of carboxyl and amide groups gives the polymer fluorescence and phosphorescence. In particular, due to the close solid accumulation of the molecular chains of PAA and PAM, which could block the entry of oxygen, and the intramolecular/intermolecular interactions such as hydrogen bonds, the cluster chromophore group is stabilized, which makes the solid of PAA and PAM exhibit significant ultra-long RTP emission under environmental conditions (Fig. 23). However, because of the introduction of isopropyl side-groups, PNIPAM solid was more permeable to oxygen in the solid, so RTP could not be observed under environmental conditions. However, RTP of PNIPAM could be observed in vacuum or under nitrogen. These experiments have proved that different lateral groups can regulate the interaction between molecules, thus affecting the photophysical properties of chromophores; these results significantly broaden the design of atypical luminescent chromophores with RTP properties. ${ }^{90}$

Yuan et al. also reported the CTE of cellulose and its derivatives in 2019. MCC, HEC, and HPC solids showed bright

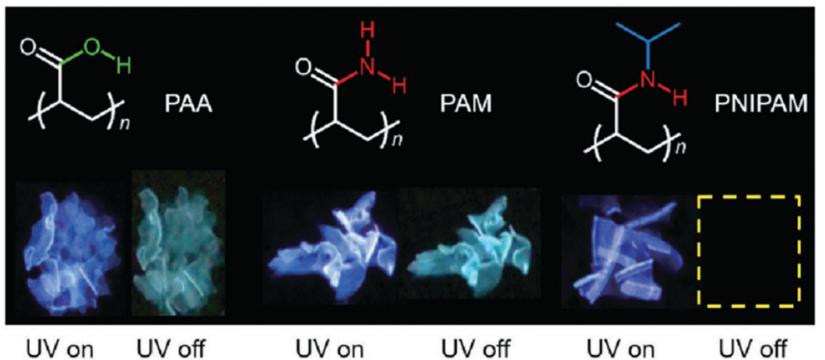

Fig. 23 Structures and photographs of the solids taken under UV light or after ceasing the UV irradiation of PAA, PAM, and PNIPAM at ambient conditions. Reprinted with permission from ref. 90. Copyright 2019, Royal Society of Chemistry.

emission alongside distinct RTP, while, CA demonstrated relatively low intensity of solid emission without noticeable RTP (Fig. 24). Their emissions were explained by the CTE mechanism and conformation rigidification. Clustering of ether, hydroxyl, and carbonyl units was responsible for the emission. Meanwhile, intramolecular and intermolecular hydrogen bond strength and solid crystallinity will affect the rigidity of the emitting cluster group, thus affecting the PL strength. The relatively weak emission of CA within this series should be ascribed to its fewer hydrogen bonds and poor crystallinity. ${ }^{91}$ It is worth mentioning that the low-temperature phosphorescence of MCC has excitation wavelength dependence, which further indicates the diversity of clustered groups in this system.

In 2020, Yuan et al. reported a clustering-triggered efficient room-temperature phosphorescence system. The effective RTP strategy in luminescent clusters is realized by the combination of lone pair clustering and effective electron interaction. The former facilitates spin-orbit coupling and subsequent ISC, while the latter reduces the energy gap and stabilizes the triplet state, thereby synergistically providing significant RTP. Remarkably, RTP from thiourea solids with unprecedentedly high efficiency of up to $24.5 \%$ is obtained. Besides, further control experiments proved the crucial role of through-space delocalization for emission (Fig. 25). ${ }^{40}$ Jin et al. reported the ultralong lifetime RTP and dual-band waveguide behavior of phosphoramidic acid oligomers (Fig. 26), with ultra-long

(a)

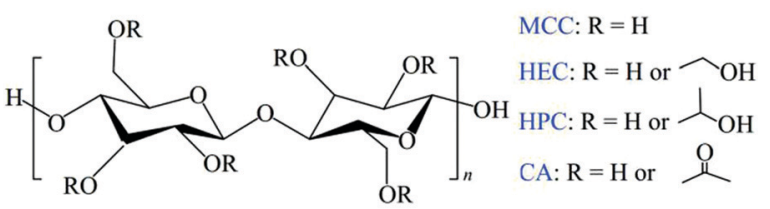

(b)

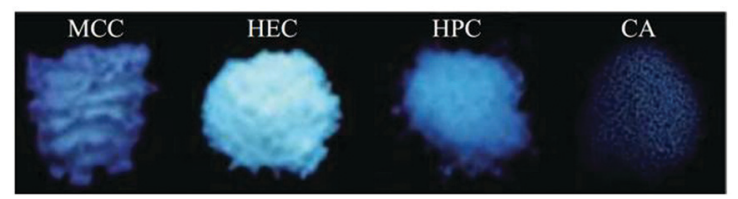

Fig. 24 (a) Chemical structures of MCC, HEC, HPC, and CA. (b) Photographs of MCC, HEC, HPC, and CA powders taken under $365 \mathrm{~nm}$ UV irradiation. Reprinted with permission from ref. 91. Copyright 2019, Springer. 
(a)
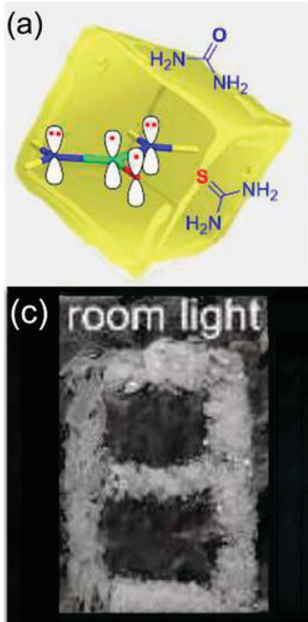

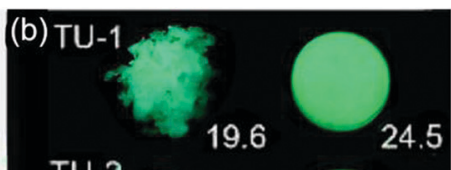

TU-2
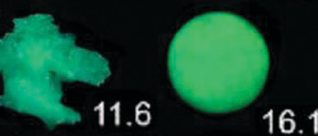

UV on

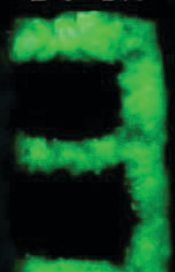

UV off

Fig. 25 (a) Structures of urea and TU and their delocalized $\pi_{6}^{4}$ system. (b) Photographs of TU-1, TU-2, and their tablets taken under $312 \mathrm{~nm}$ UV light. $\Phi$ values (\%) of the samples are also given. (c) Pattern " 8 " comprising urea (blue part) and TU-1 (green part) crystals taken under room light, $312 \mathrm{~nm}$ UV light, and after ceasing the UV irradiation. Reprinted with permission from ref. 40. Copyright 2020, John Wiley and Sons.

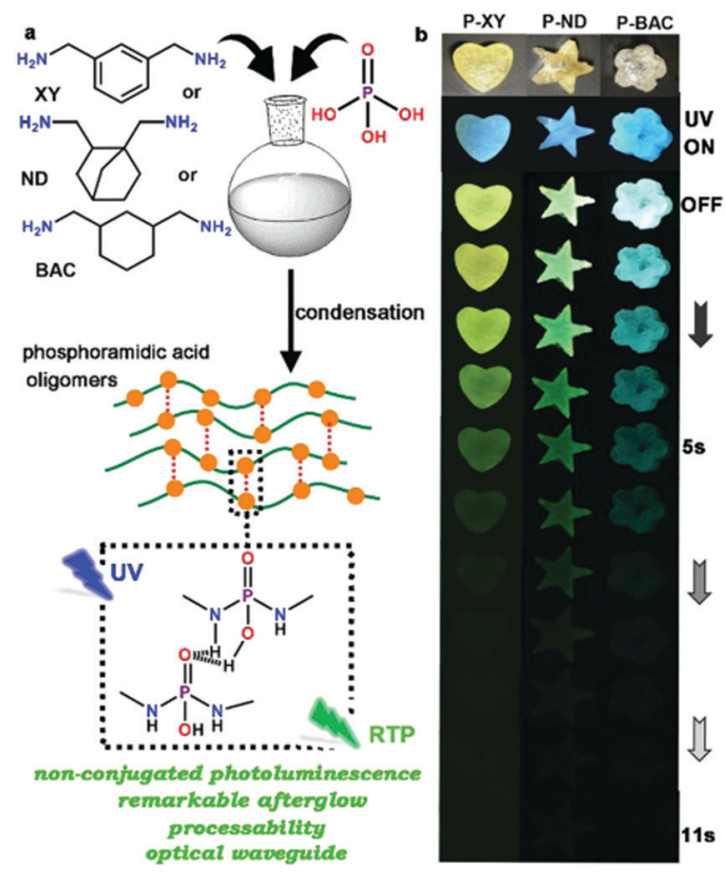

Fig. 26 (a) Schematic illustration of polycondensation reaction between phosphoric acid and aryl/alkyl diamine, and possible local ordered assembly behavior to form a local rigid structure of three phosphoramidic acid oligomers. (The orange spheres represent phosphoramidic acid groups and the red dashed lines represent inter-chain hydrogen bonds.) (b) The photographs of three oligomers under daylight or UV-365 nm radiation, and the long afterglow after ceasing UV-365 nm radiation in an oxygen environment at room temperature. Reprinted with permission from ref. 92. Copyright 2020, Royal Society of Chemistry.

phosphorescence lifetimes (376.5 ms and $776.9 \mathrm{~ms}$ ) and high phosphorescence PLQYs (4.6-10.5\%). ${ }^{92}$

\section{Summary and outlook}

To sum up, atypical luminescent chromophores have attracted wide attention and various types of them have been reported. Because these chromophores are mainly composed of aliphatic chains, amine, amide, ester, carbonyl and other similar structural units, their chemical composition is closer to biological chromophores such as proteins and polysaccharides than to the typical conjugated luminescent chromophores. These atypical chromophores are endowed with superior properties such as better hydrophilicity, lower cytotoxicity, eco-friendliness and excellent chain flexibility. There is now convincing evidence that the emission and/or the tunability of unconventional chromophores does not rely entirely on some specific aggregation or conformation, but upon the interactions among chromophores with heterogeneity and complexity. Besides, efficient throughspace conjugation and conformational rigidification is also responsible for the color-tunable PL and/or persistent RTP.

Future priorities for research are: (i) to explore physical images and high-level theoretical calculations of the interactions among these atypical chromophore groups, and (ii) to synthesize and study the structure-activity relationships of new luminescent materials. These endeavors will not only play an important role in clarifying the mechanism of PL and avoid some one-sided views, but will also develop new materials that are convenient, inexpensive and environmentally friendly. Future innovations can be expected to include: (i) simple and controllable preparation routes for materials with ultra-long PL/RTP lifetime; (ii) in-depth understanding of their emission mechanisms; (iii) obtaining materials with higher PLQYs, especially at longer wavelengths; (iv) new mechanoresponsive materials with color-tunable PL and/or persistent RTP.

\section{Conflicts of interest}

There are no conflicts to declare.

\section{Acknowledgements}

The work was funded by NSFC (No. 51473028), the key scientific and technological project of Jilin province (20150204011GX, 20160307016GX, 20190701010GH), the Development and Reform Commission of Jilin province (20160058, 2020C035-5). Open Project of State Key Laboratory of Supramolecular Structure and Materials (sklssm202039). M. R. B. thanks EPSRC grant $\mathrm{EL} / \mathrm{L} 02621 \mathrm{X} / 1$ for funding.

\section{Notes and references}

1 J. Liu, Y. Cheng, Z. Xie, Y. Geng, L. Wang, X. Jing and F. Wang, White Electroluminescence from a Star-like Polymer with an Orange Emissive Core and Four Blue Emissive Arms, Adv. Mater., 2008, 20, 1357-1362.

2 M. Fang, J. Yang, X. Xiang, Y. Xie, Y. Dong, Q. Peng, Q. Li and $\mathrm{Z}$. Li, Unexpected room-temperature phosphorescence from a non-aromatic, low molecular weight, pure organic 
molecule through the intermolecular hydrogen bond, Mater. Chem. Front., 2018, 2, 2124-2129.

3 G. Zhou, W.-Y. Wong, B. Yao, Z. Xie and L. Wang, Triphenylamine-Dendronized Pure Red Iridium Phosphors with Superior OLED Efficiency/Color Purity Trade-Offs, Angew. Chem., Int. Ed., 2007, 46, 1149-1151.

4 Q. Li, Y. Tang, W. Hu and Z. Li, Fluorescence of nonaromatic organic systems and room temperature phosphorescence of organic luminogens: The intrinsic principle and recent progress, Small, 2018, 14, 1801560.

5 Z. Mao, Z. Yang, C. Xu, Z. Xie, L. Jiang, F. L. Gu, J. Zhao, Y. Zhang, M. P. Aldred and Z. Chi, Two-photon-excited ultralong organic room temperature phosphorescence by dual-channel triplet harvesting, Chem. Sci., 2019, 10, 7352-7357.

6 W. Wu, R. Tang, Q. Li and Z. Li, Functional hyperbranched polymers with advanced optical, electrical and magnetic properties, Chem. Soc. Rev., 2015, 44, 3997-4022.

7 Q. Wang, J. Ding, D. Ma, Y. Cheng, L. Wang, X. Jing and F. Wang, Harvesting Excitons Via Two Parallel Channels for Efficient White Organic LEDs with Nearly 100\% Internal Quantum Efficiency: Fabrication and Emission-Mechanism Analysis, Adv. Funct. Mater., 2009, 19, 84-95.

8 G. Li, D. Zhu, X. Wang, Z. Su and M. R. Bryce, Dinuclear metal complexes: multifunctional properties and applications, Chem. Soc. Rev., 2020, 49, 765-838.

9 J. Yang, Z. Ren, Z. Xie, Y. Liu, C. Wang, Y. Xie, Q. Peng, B. Xu, W. Tian, F. Zhang, Z. Chi, Q. Li and Z. Li, AIEgen with fluorescence-phosphorescence dual mechanoluminescence at room temperature, Angew. Chem., Int. Ed., 2017, 129, 898-902.

10 M. Du, Y. Feng, D. Zhu, T. Peng, Y. Liu, Y. Wang and M. R. Bryce, Novel emitting system based on a multifunctional bipolar phosphor: an effective approach for highly efficient warm-white light-emitting devices with high color-rendering index at high luminance, Adv. Mater., 2016, 28, 5963-5968.

11 J. Yang, Z. Ren, B. Chen, M. Fang, Z. Zhao, B. Z. Tang, Q. Peng and Z. Li, Three polymorphs of one luminogen: how the molecular packing affects the RTP and AIE properties?, J. Mater. Chem. C, 2017, 5, 9242-9246.

12 J. Mei, N. L. C. Leung, R. T. K. Kwok, J. W. Y. Lam and B. Z. Tang, Aggregation-Induced Emission: Together We Shine, United We Soar!, Chem. Rev., 2015, 115, 11718-11940.

13 R. Hu, N. L. C. Leung and B. Z. Tang, AIE macromolecules: syntheses, structures and functionalities, Chem. Soc. Rev., 2014, 43, 4494-4562.

14 Y. Gong, L. Zhao, Q. Peng, D. Fan, W. Z. Yuan, Y. Zhang and B. Z. Tang, Crystallization-induced dual emission from metaland heavy atom-free aromatic acids and esters, Chem. Sci., 2015, 6, 4438-4444.

15 D. Dang, Z. Qiu, T. Han, Y. Liu, M. Chen, R. T. K. Kwok, J. W. Y. Lam and B. Z. Tang, $1+1 \gg 2$ : Dramatically Enhancing the Emission Efficiency of TPE-Based AIEgens but Keeping their Emission Color through Tailored Alkyl Linkages, Adv. Funct. Mater., 2018, 28, 1707210.

16 J. Zhang, B. Xu, J. Chen, S. Ma, Y. Dong, L. Wang, B. Li, L. Ye and W. Tian, An Organic Luminescent Molecule: What Will
Happen When the "Butterflies" Come Together?, Adv. Mater., 2014, 26, 739-745.

17 Y. Kawamura, J. Brooks, J. J. Brown, H. Sasabe and C. Adachi, Intermolecular Interaction and a Concentration-Quenching Mechanism of Phosphorescent Ir(III) Complexes in a Solid Film, Phys. Rev. Lett., 2006, 96, 017404.

18 J. Mei, Y. Hong, J. W. Lam, A. Qin, Y. Tang and B. Z. Tang, Aggregation-induced emission: the whole is more brilliant than the parts, Adv. Mater., 2014, 26, 5429-5479.

19 J. Luo, Z. Xie, J. W. Y. Lam, L. Cheng, H. Chen, C. Qiu, H. S. Hwok, X. Zhan, Y. Liu, D. Zhu and B. Z. Tang, Aggregationinduced emission of 1-methyl-1,2,3,4,5-pentaphenylsilole, Chem. Commun., 2001, 1740-1741.

20 R. B. Restani, P. I. Morgado, M. P. Ribeiro, I. J. Correia, A. Aguiar-Ricardo and V. D. Bonifacio, Biocompatible Polyurea Dendrimers with pH-Dependent Fluorescence, Angew. Chem., Int. Ed., 2012, 51, 5162-5165.

21 T. Huang, Z. Wang, A. Qin, Z. J. Sun and B. Z. Tang, Luminescent Polymers Containing Unconventional Chromophores, Acta Chim. Sin., 2013, 71, 979-990.

22 R. B. Wang, W. Z. Yuan and X. Y. Zhu, Aggregation-induced Emission of Non-conjugated Poly(amido amine)s: Discovering, Luminescent Mechanism Understanding and Bioapplication, Chin. J. Polym. Sci., 2015, 33, 680-687.

23 S. Cai, H. Ma, H. Shi, H. Wang, X. Wang, L. Xiao, W. Ye, K. Huang, X. Cao, N. Gan, C. Ma, M. Gu, L. Song, H. Xu, Y. Tao, C. Zhang, W. Yao, Z. An and W. Huang, Enabling long-lived organic room temperature phosphorescence in polymers by subunit interlocking, Nat. Commun., 2019, 10, 4247-4255.

24 H. Chen, X. Yao, X. Ma and H. Tian, Amorphous, Efficient, Room-Temperature Phosphorescent Metal-Free Polymers and Their Applications as Encryption Ink, Adv. Opt. Mater., 2016, 4, 1397-1401.

25 N. Jiang, D. Zhu, Z. Su and M. R. Bryce, Blue-emitting thermoreversible oligourethane gelators with aggregationinduced emission properties, J. Mater. Chem. C, 2020, 8, 5137-5142.

26 X. Bin, W. Luo, W. Yuan and Y. Zhang, Clustering-Triggered Emission of Poly(N-hydroxysuccinimide Methacrylate), Acta Chim. Sin., 2016, 74, 935-942.

27 J. J. Yan, X. Y. Wang, M. Z. Wang, D. H. Pan, R. L. Yang, Y. P. Xu, L. Z. Wang and M. Yang, Self-Assembling Nonconjugated Poly(amide-imide) into Thermoresponsive Nanovesicles with Unexpected Red Fluorescence for Bioimaging, Biochromophores, 2019, 20, 1455-1463.

28 B. Saha, B. Ruidas, S. Mete, C. D. Mukhopadhyay, K. Bauri and P. De, AIE-active non-conjugated poly(Nvinylcaprolactam) as a fluorescent thermometer for intracellular temperature imaging, Chem. Sci., 2020, 11, 141-147.

29 N. Jiang, G. Li, W. Che, D. Zhu, Z. Su and M. R. Bryce, Polyurethane derivatives for highly sensitive and selective fluorescence detection of 2,4,6-trinitrophenol (TNP), J. Mater. Chem. C, 2018, 6, 11287-11291.

30 K. Bauri, B. Saha, J. Mahanti and P. De, A nonconjugated macromolecular luminogen for speedy, selective and sensitive 
detection of picric acid in water, Polym. Chem., 2017, 8, 7180-7187.

31 J. Yang, Y. Zhang, S. Gautama, L. Liu, J. Deya, W. Chen, R. P. Mason, C. A. Serranoe, K. A. Schuge and L. Tang, Development of aliphatic biodegradable photoluminescent polymers, Proc. Natl. Acad. Sci. U. S. A., 2009, 106, 10086-10091.

32 Q. Miao, C. Xie, X. Zhen, Y. Lyu, H. Duan, X. Liu, J. V. Jokerst and $\mathrm{K} . \mathrm{Pu}$, Molecular afterglow imaging with bright, biodegradable polymer nanoparticles, Nat. Biotechnol., 2017, 35, 1102-1110.

33 J. A. Mikroyannidis and I. K. Spiliopoulos, Synthesis and Optical Properties of Novel Blue-Light-Emitting Poly $(p$ phenylene vinylene) Derivatives with Pendant Oxadiazole or Cyano Groups, J. Polym. Sci., Part A: Polym. Chem., 2004, 42, 1768-1778.

34 T. Liu, Y. Meng, X. Wang, H. Wang and X. Li, Unusual strong fluorescence of a hyperbranched phosphate: discovery and explanations, $R S C A d v ., 2013,3$, 8269-8275.

35 W. Li, X. Wu, Z. Zhao, A. Qin, R. Hu and B. Z. Tang, CatalystFree, Atom-Economic, Multicomponent Polymerizations of Aromatic Diynes, Elemental Sulfur, and Aliphatic Diamines toward Luminescent Polythioamides, Macromolecules, 2015, 48, 7747-7754.

36 J.-J. Yan, Z.-K. Wang, X.-S. Lin, C.-Y. Hong, H.-J. Liang, C.-Y. Pan and Y.-Z. You, Polymerizing Nonfluorescent Monomers without Incorporating any Fluorescent Agent Produces Strong Fluorescent Polymers, Adv. Mater., 2012, 24, 5617-5624.

37 Z. Zhao, X. Chen, Q. Wang, T. Yang, Y. Zhang and W. Z. Yuan, Sulphur-containing nonaromatic polymers: clusteringtriggered emission and luminescence regulation by oxidation, Polym. Chem., 2019, 10, 3639-3646.

38 W. Yu, Y. Wu, J. Chen, X. Duan, X.-F. Jiang, X. Qiu and Y. Li, Sulfonated ethylenediamine-acetone-formaldehyde condensate: preparation, unconventional photoluminescence and aggregation enhanced emission, RSC Adv., 2016, 6, 51257-51263.

39 Q. Zhou, B. Cao, C. Zhu, S. Xu, Y. Gong, W. Z. Yuan and Y. Zhang, Clustering-Triggered Emission of Nonconjugated Polyacrylonitrile, Small, 2016, 12, 6586-6592.

40 S. Zheng, T. Hu, X. Bin, Y. Wang, Y. Yi, Y. Zhang and W. Z. Yuan, Clustering-Triggered Efficient Room-Temperature Phosphorescence from Nonconventional Luminophores, ChemPhysChem, 2020, 21, 36-42.

41 C. Deng, X. Chen, H. Yu, J. Sun, T. Lu and X. Jing, A biodegradable triblock copolymer poly(ethylene glycol)- $b$ poly(L-lactide)- $b$-poly(L-lysine): Synthesis, self-assembly, and RGD peptide modification, Polymer, 2007, 48, 139-149.

42 C. C. Chu and T. M. Imae, Fluorescence Investigations of Oxygen-Doped Simple Amine Compared with Fluorescent PAMAM Dendrimer, Macromol. Rapid Commun., 2009, 30, 89-93.

43 S.-F. Shiau, T.-Y. Juang, H.-W. Chou and M. Liang, Synthesis and properties of new water-soluble aliphatic hyperbranched poly(amido acids) with high $\mathrm{pH}$-dependent photoluminescence, Polymer, 2013, 54, 623-630.

44 S. Bhattacharya, V. N. Rao, S. Sarkar and R. Shunmugam, Unusual emission from norbornene derived phosphonate molecule-a sensor for $\mathrm{Fe}^{\mathrm{III}}$ in aqueous environment, Nanoscale, 2012, 4, 6962-6966.

45 X. Chen, X. Liu, J. Lei, L. Xu, Z. Zhao, F. Kausar, X. Xie, X. Zhu, Y. Zhang and W. Z. Yuan, Synthesis, clusteringtriggered emission, explosive detection and cell imaging of nonaromatic polyurethanes, Mol. Syst. Des. Eng., 2018, 3, 364-375.

46 L. Yang, L. Wang, C. Cui, J. Lei and J. Zhang, Stöber strategy for synthesizing multifluorescent organosilica nanocrystals, Chem. Commun., 2016, 52, 6154-6157.

47 Y. Wang, X. Bin, X. Chen, S. Zheng, Y. Zhang and W. Z. Yuan, Emission and Emissive Mechanism of Nonaromatic Oxygen Clusters, Macromol. Rapid Commun., 2018, 39, 1800528.

48 X. Miao, T. Liu, C. Zhang, X. Geng, Y. Meng and X. Li, Fluorescent aliphatic hyperbranched polyether: chromophorefree and without any N and P atoms, Phys. Chem. Chem. Phys., 2016, 18, 4295-4299.

49 Q. Zhang, Q. Mao, C. Shang, Y. N. Chen, X. Peng, H. Tan and H. Wang, Simple aliphatic oximes as nonconventional luminogens with aggregation-induced emission characteristics, J. Mater. Chem. C, 2017, 5, 3699-3705.

50 M. Sun, C. Y. Hong and C. Y. Pan, A Unique Aliphatic Tertiary Amine Chromophore: Fluorescence, Polymer Structure, and Application in Cell Imaging, J. Am. Chem. Soc., 2012, 134, 20581-20584.

51 D. Wang and T. Imae, Fluorescence Emission from Dendrimers and Its $\mathrm{pH}$ Dependence, J. Am. Chem. Soc., 2004, 126, 13204-13205.

52 W. I. Lee, Y. Bae and A. J. Bard, Strong Blue Photoluminescence and ECL from OH-Terminated PAMAM Dendrimers in the Absence of Gold Nanoparticles, J. Am. Chem. Soc., 2004, 126, 8358-8359.

53 L. Pastor-Pérez, Y. Chen, Z. Shen, A. Lahoz and S. E. Stiriba, Unprecedented Blue Intrinsic Photoluminescence from Hyperbranched and Linear Polyethylenimines: Polymer Architectures and pH-Effects, Macromol. Rapid Commun., 2007, 28, 1404-1409.

54 L. Cao, W. Yang, C. Wang and S. Fu, Synthesis and Striking Fluorescence Properties of Hyperbranched Poly(amido amine), J. Macromol. Sci., Part A: Pure Appl. Chem., 2007, 44, 417-424.

55 A. Shukla, S. Mukherjee, S. Sharma, V. Agrawal, K. K. V. Radha and P. Guptasarma, A novel UV laser-induced visible blue radiation from protein crystals and aggregates: scattering artifacts or fluorescence transitions of peptide electrons delocalized through hydrogen bonding?, Arch. Biochem. Biophys., 2004, 428, 144-153.

56 C. M. Xing, J. W. Y. Lam, A. Qin, Y. Dong, M. Haußler, W. T. Yang and B. Z. Tang, Unique photoluminescence from nonconjugated alternating copolymer poly[(maleic anhydride)alt-(vinyl acetate)], Polym. Mater. Sci. Eng, 2007, 96, 418-419.

57 M.-M. Fang, J. Yang and Z. Li, Recent Advances in Purely Organic Room Temperature Phosphorescence Polymer, Chin., J. Polym. Sci., 2019, 37, 383-393.

58 Y. Gong, Y. Tan, J. Mei, Y. Zhang, W. Z. Yuan, J. Z. Sun and B. Z. Tang, Room temperature phosphorescence from 
natural products: Crystallization matters, Sci. China: Chem., 2013, 56, 1178-1182.

59 J. Yang, X. Zhen, B. Wang, X. Gao, Z. Ren, J. Wang, J. Xie, J. Li, Q. Peng, K. Pu and Z. Li, The influence of the molecular packing on the room temperature phosphorescence of purely organic luminogens, Nat. Commun., 2018, 9, 840-850.

60 M. Shimizu, T. Kinoshita, R. Shigitani, Y. Miyake and K. Tajima, Use of silylmethoxy groups as inducers of efficient room temperature phosphorescence from preciousmetal-free organic luminophores, Mater. Chem. Front., 2018, 2, 347-354.

61 Y. Xie and Z. Li, Thermally activated delayed fluorescent polymers, J. Polym. Sci., Part A: Polym. Chem., 2017, 55, 575-584.

62 K. Li, L. Zhao, Y. Gong, W. Yuan and Y. Zhang, A gelable pure organic luminogen with fluorescence-phosphorescence dual emission, Sci. China: Chem., 2017, 60, 806-812.

63 J. Yang, X. Gao, Z. Xie, Y. Gong, M. Fang, Q. Peng, Z. Chi and $\mathrm{Z}$. Li, Elucidating the excited state of mechanoluminescence in organic luminogens with room-temperature phosphorescence, Angew. Chem., Int. Ed., 2017, 56, 15299-15303.

64 Z. Chai, C. Wang, J. Wang, F. Liu, Y. Xie, Y.-Z. Zhang, J.-R. Li, Q. Li and Z. Li, Abnormal room temperature phosphorescence of purely organic boron-containing compounds: the relationship between the emissive behavior and the molecular packing, and the potential related applications, Chem. Sci., 2017, 8, 8336-8344.

65 Y. Xie, Y. Ge, Q. Peng, C. Li, Q. Li and Z. Li, How the molecular packing affects the room temperature phosphorescence in pure organic compounds: Ingenious molecular design, detailed crystal analysis, and rational theoretical calculations, Adv. Mater., 2017, 1606829.

66 Y. Ruff and J.-M. Lehn, Glycodynamers: Fluorescent Dynamic Analogues of Polysaccharides, Angew. Chem., Int. Ed., 2008, 47, 3556-3559.

67 O. Varnavski, R. G. Ispasoiu, L. Balogh, D. Tomalia and T. Goodson, Ultrafast time-resolved photoluminescence from novel metal-dendrimer nanocomposites, J. Chem. Phys., 2001, 114, 1962-1965.

68 G. A. Crosby and J. N. Demas, Measurement of photoluminescence quantum yields, Review, J. Phys. Chem., 1971, 75, 991-1024.

69 J. Zheng, J. T. Petty and R. M. Dickson, High Quantum Yield Blue Emission from Water-Soluble $\mathrm{Au}_{8}$ Nanodots, J. Am. Chem. Soc., 2003, 125, 7780-7781.

70 D. Wang, T. Imae and M. Miki, Fluorescence emission from PAMAM and PPI dendrimers, J. Colloid Interface Sci., 2007, 306, 222-227.

71 S.-Y. Lin, T.-H. Wu, Y.-C. Jao, C.-P. Liu, H.-Y. Lin, L.-W. Lo and C.-S. Yang, Unraveling the Photoluminescence Puzzle of PAMAM Dendrimers, Chem. - Eur. J., 2011, 17, 7158-7161.

72 W. Yang and C.-Y. Pan, Synthesis and Fluorescent Properties of Biodegradable Hyperbranched Poly(amido amine)s, Macromol. Rapid Commun., 2009, 30, 2096-2101.

73 W. Yang, C.-Y. Pan, M.-D. Luo and H.-B. Zhang, Fluorescent Mannose-Functionalized Hyperbranched Poly(amido amine)s:
Synthesis and Interaction with E. coli, Biomacromolecules, 2010, 11, 1840-1846.

74 S. Niu, H. Yan, Z. Chen, S. Li, P. Xu and X. Zhi, Unanticipated bright blue fluorescence produced from novel hyperbranched polysiloxanes carrying unconjugated carbon-carbon double bonds and hydroxyl groups, Polym. Chem., 2016, 7, 3747-3755.

75 R. Ye, Y. Liu, H. Zhang, H. Su, Y. Zhang, L. Xu, R. Hu, R. T. K. Kwok, K. S. Wong, J. W. Y. Lam, W. A. Goddard and B. Z. Tang, Non-conventional fluorescent biogenic and synthetic polymers without aromatic rings, Polym. Chem., 2017, 8, 1722-1727.

76 E. Zhao, J. W. Y. Lam, L. Meng, Y. Hong, H. Deng, G. Bai, X. Huang, J. Hao and B. Z. Tang, Poly[(maleic anhydride)alt-(vinyl acetate)]: A Pure Oxygenic Nonconjugated Macromolecule with Strong Light Emission and Solvatochromic Effect, Macromolecules, 2015, 48, 64-71.

77 A. Pucci, R. Rausa and F. Ciardelli, Aggregation-Induced Luminescence of Polyisobutene Succinic Anhydrides and Imides, Macromol. Chem. Phys., 2008, 209, 900-906.

78 W. Yu, Z. Wang, D. Yang, X. Ouyang, X. Qiu and Y. Li, Nonconventional photoluminescence from sulfonated acetone-formaldehyde condensate with aggregation-enhanced emission, RSC Adv., 2016, 6, 47632-47636.

79 W. Z. Yuan and Y. Zhang, Nonconventional Macromolecular Luminogens with Aggregation-Induced Emission Characteristics, J. Polym. Sci., Part A: Polym. Chem., 2017, 55, 560-574.

80 X. Zhou, W. Luo, H. Nie, L. Xu, R. Hu, Z. Zhao, A. Qin and B. Z. Tang, Oligo(maleic anhydride)s: a platform for unveiling the mechanism of clusteroluminescence of non-aromatic polymers, J. Mater. Chem. C, 2017, 5, 4775-4779.

81 X. Chen, Z. He, F. Kausar, G. Chen, Y. Zhang and W. Z. Yuan, Aggregation-Induced Dual Emission and Unusual Luminescence beyond Excimer Emission of Poly(ethylene terephthalate), Macromolecules, 2018, 51, 9035-9042.

82 Y. Feng, T. Bai, H. Yan, F. Ding, L. Bai and W. Feng, High Fluorescence Quantum Yield Based on the Through-Space Conjugation of Hyperbranched Polysiloxane, Macromolecules, 2019, 52, 3075-3082.

83 C. Shang, N. Wei, H. Zhuo, Y. Shao, Q. Zhang, Z. Zhang and H. Wang, Highly emissive poly(maleic anhydride-alt-vinyl pyrrolidone) with molecular weight-dependent and excitationdependent fluorescence, J. Mater. Chem. C, 2017, 5, 8082-8090.

84 C. Hu, Y. Ru, Z. Guo, Z. Liu, J. Song, W. Song, X. Zhang and J. Qiao, New multicolored AIE photoluminescent polymers prepared by controlling the $\mathrm{pH}$ value, J. Mater. Chem. C, 2019, 7, 387-393.

85 L. Yuan, H. Yan, L. Bai, T. Bai, Y. Zhao, L. Wang and Y. Feng, Unprecedented Multicolor Photoluminescence from Hyperbranched Poly(amino ester)s, Macromol. Rapid Commun., 2019, 40, 1800658.

86 L. Fang, C. Huang, G. Shabir, J. Liang, Z. Liu and H. Zhang, Hyperbranching-Enhanced-Emission Effect Discovered in Hyperbranched Poly(4-(cyanomethyl)phenyl methacrylate), ACS Macro. Lett., 2019, 8, 1605-1610.

87 Z. Zhang, J. Xiong, G. He, D. Dang, Y. Xie and Q. Wang, Fluorous effect-induced emission of azido substituted 
poly(vinylidene fluoride) with high photostability and film formation, Polym. Chem., 2020, 11, 1307-1313.

88 N. Jiang, G.-F. Li, B.-H. Zhang, D.-X. Zhu, Z.-M. Su and M. R. Bryce, Aggregation-Induced Long-Lived Phosphorescence in Nonconjugated Polyurethane Derivatives at $77 \mathrm{~K}$, Macromolecules, 2018, 51, 4178-4184.

89 X. Chen, W. Luo, H. Ma, Q. Peng, W. Z. Yuan and Y. Zhang, Prevalent intrinsic emission from nonaromatic amino acids and poly(amino acids), Sci. China: Chem., 2018, 61, 351-359.

90 Q. Zhou, Z. Wang, X. Dou, Y. Wang, S. Liu, Y. Zhang and W. Z. Yuan, Emission mechanism understanding and tunable persistent room temperature phosphorescence of amorphous nonaromatic polymers, Mater. Chem. Front., 2019, 3, 257-264.

91 L. L. Du, B. L. Jiang, X. H. Chen, Y. Z. Wang, L. M. Zou, Y. L. Liu, Y. Y. Gong, C. Wei and W. Z. Yuan, Clustering-triggered Emission of Cellulose and Its Derivatives, Chin. J. Polym. Sci., 2019, 37, 409-415.

92 Z.-F. Liu, X. Chen and W. J. Jin, Ultralong lifetime room temperature phosphorescence and dual-band waveguide behavior of phosphoramidic acid oligomers, J. Mater. Chem. C, 2020, 8, 7330-7335. 